KANAZAWA-11-07

SHEP-11-06

UT-HET 052

\title{
Light Charged Higgs bosons at the LHC in 2HDMs
}

\author{
Mayumi Aoki ${ }^{1 *}$, Renato Guedes ${ }^{2} \dagger$, Shinya Kanemura ${ }^{3} \ddagger$ \\ Stefano Moretti ${ }^{4}$ \&, Rui Santos ${ }^{2,5}$ ๆ and Kei Yagyu ${ }^{3}$ ** \\ ${ }^{1}$ Institute for Theoretical Physics, Kanazawa University, Kanazawa 920-1192, Japan. \\ ${ }^{2}$ Centro de Física Teórica e Computacional, \\ Faculdade de Ciências, Universidade de Lisboa, \\ Av. Prof. Gama Pinto 2, 1649-003 Lisboa, Portugal. \\ ${ }^{3}$ Department of Physics, The University of Toyama, 3190 Gofuku, Toyama 930-8555, Japan. \\ ${ }^{4}$ NExT Institute and School of Physics and Astronomy, \\ University of Southampton, Highfield, Southampton SO17 1BJ, UK. and \\ ${ }^{5}$ Instituto Superior de Engenharia de Lisboa, \\ Rua Conselheiro Emídio Navarro 1, 1959-007 Lisboa, Portugal
}

(Dated: June 15, 2018)

\begin{abstract}
We present a discussion of light charged Higgs boson searches at the Large Hadron Collider (LHC) in CP-conserving 2-Higgs Doublet Models (2HDMs). Taking into account all available experimental and theoretical constraints we review all possible processes that would allow for a detection of such a particle with a mass below the top quark mass. Two different types of processes are analysed: one that depends only on $\tan \beta$ and on the charged Higgs boson mass because it involves only the charged Higgs boson Yukawa couplings; the other that depends on almost all model parameters, mainly due to the presence of Higgs self-couplings. We discuss the regions of parameter space of $2 \mathrm{HDMs}$ that can be covered by each type of process and define some guidelines for experimental searches at the LHC.
\end{abstract}

\section{INTRODUCTION}

The Large Hadron Collider ( $\mathrm{LHC}$ ) is operating at a Centre-of-Mass (CM) energy of $7 \mathrm{TeV}$. Soon it will start operating at an energy of $14 \mathrm{TeV}$. One of the most striking evidences of physics Beyond the Standard Model (BSM) would be the appearance of a charged Higgs boson, $H^{ \pm}$. As light charged Higgs particles, for which $m_{H^{ \pm}}<m_{t}$ (the top mass), are simpler to detect, it is timely to perform a study on the possible models where these states are still allowed. The LEP experiments have set a lower limit on the mass of a charged Higgs boson, of $79.3 \mathrm{GeV}$ at $95 \%$ Confidence Level (CL), assuming that $\mathrm{BR}\left(H^{+} \rightarrow \tau^{+} \nu\right)+B R\left(H^{+} \rightarrow c \bar{s}\right)=1$ holds for the possible charged Higgs boson Branching Ratios (BRs) [1]. This limit becomes stronger if $\operatorname{BR}\left(H^{+} \rightarrow \tau^{+} \nu\right) \approx 1$ (see [2] for a discussion). Searches at the Tevatron [3] based on $t \bar{t}$ production with the top decaying via $t \rightarrow b H^{+}$and assuming $\mathrm{BR}\left(H^{+} \rightarrow \tau^{+} \nu\right) \approx 1$ have yielded a limit of $\mathrm{BR}\left(t \rightarrow b H^{+}\right)<0.2$ for a charged Higgs mass of $100 \mathrm{GeV}$. Indeed, all models discussed in this work have a $\operatorname{BR}\left(t \rightarrow b H^{+}\right)$below the Tevatron limit when $\operatorname{BR}\left(H^{+} \rightarrow \tau^{+} \nu\right) \approx 1^{1}$. In this study we will concentrate on the $14 \mathrm{TeV} \mathrm{CM}$ energy because, as it will become clear later on, not only the cross sections grow with energy but also a significant luminosity is needed to start probing these models. We therefore defer the study of the $7 \mathrm{TeV}$ case to a separate publication.

The simplest extensions of the SM that give rise to charged Higgs bosons amount to the addition of an extra $S U(2)$ Higgs doublet to the SM field content. The most common CP-conserving 2HDM has a softly broken $Z_{2}$ symmetry. When this symmetry is extended to the fermions to avoid Flavour Changing

\footnotetext{
* mayumi@hep.s.kanazawa-u.ac.jp

$\dagger$ renato@cii.fc.ul.pt

¥ kanemu@sci.u-toyama.ac.jp

$\S$ stefano@phys.soton.ac.uk

I rsantos@cii.fc.ul.pt

** keiyagyu@jodo.sci.u-toyama.ac.jp

1 This conclusion already takes into account other experimental constraints.
} 
Neutral Currents (FCNCs) we end up with four [4] different models, to be described in detail later, which we will call Type I, Type II, Type Y [5] and Type X [5] (named I, II, III and IV in [4], respectively). Constraints from $B$-physics, and particularly those coming from $b \rightarrow s \gamma$ []] , have excluded a charged Higgs boson with a mass below approximately $300 \mathrm{GeV}$ almost independently of $\tan \beta=v_{2} / v_{1}$ - the ratio of the Vacuum Expectation Values (VEVs) of the two doublets - in models Type II and Type Y. Charged Higgs bosons with masses as low as $100 \mathrm{GeV}$ are instead still allowed in models Type I and X [2, 5, 7]. The models as well as their experimental and theoretical constraints will be discussed in detail in the next section.

It is important to note that the phenomenology of a light charged Higgs boson, to be discussed in this work for charged Higgs searches in specific $2 \mathrm{HDM}$ models, is much more general. There is in fact a number of models that share a common charged Higgs boson phenomenology for vast regions of the parameter space of those discussed here. All such models have in common the fact they have a specific type of 2HDM as submodel for Electro-Weak Symmetry Breaking (EWSB). Recently, a number of these scenarios have been discussed in the literature [8], wherein the charged Higgs boson BR into leptons is enhanced relative to the SM case (Type X). These models provide Dark Matter (DM) candidates naturally and can accommodate neutrino oscillations and the strong first order phase transition required for successful baryogenesis while being in agreement with all experimental data.

The plan of the paper is as follows. The next section is devoted to describe the $2 \mathrm{HDM}$ versions we are using and their Yukawa Types together with the necessary conditions for the existence of a light charged Higgs boson. Section III describes the main production and decay modes of such a light Higgs state at the LHC. Each subsection of section III is devoted to the analysis of a specific production process. In Section IV we discuss the benchmarks for the search of a light charged Higgs boson at the LHC and draw our conclusions. Finally, one Appendix has the detailed description of the parton level analysis of charged Higgs bosons coming from single top production processes; a second Appendix details our use of results on charged Higgs boson pair production in left-right symmetric models for the purpose of studying the 2HDMs considered here.

\section{THE FLAVOUR CONSERVING 2HDM}

We start with a review of the basic $2 \mathrm{HDM}$ used in this work. The $2 \mathrm{HDM}$ potential chosen here is the most general, renormalisable and invariant under $S U(2) \otimes U(1)$ that one can build with two complex $S U(2)$ Higgs doublets with a softly broken $Z_{2}$ symmetry. It can be written as

$$
\begin{aligned}
V_{2 \mathrm{HDM}}= & m_{1}^{2}\left|\Phi_{1}\right|^{2}+m_{2}^{2}\left|\Phi_{2}\right|^{2}-\left(m_{3}^{2} \Phi_{1}^{\dagger} \Phi_{2}+\text { h.c. }\right) \\
& +\frac{1}{2} \lambda_{1}\left|\Phi_{1}\right|^{4}+\frac{1}{2} \lambda_{2}\left|\Phi_{2}\right|^{4}+\lambda_{3}\left|\Phi_{1}\right|^{2}\left|\Phi_{2}\right|^{2}+\lambda_{4}\left|\Phi_{1}^{\dagger} \Phi_{2}\right|^{2}+\frac{\lambda_{5}}{2}\left\{\left(\Phi_{1}^{\dagger} \Phi_{2}\right)^{2}+\text { h.c. }\right\},
\end{aligned}
$$

where $m_{i}^{2}, i=1,2,3$ and $\lambda_{i}$ are real (we assume CP conservation). The Higgs doublet fields' VEVs are $v_{1}$ and $v_{2}$ and satisfy $v_{1}^{2}+v_{2}^{2}=v^{2} \simeq(246 \mathrm{GeV})^{2}$. As CP is conserved, and once the $S U(2)$ symmetry is broken, we end up with two CP-even Higgs states, $h$ and $H$, one CP-odd state, $A$, two charged Higgs bosons, $H^{ \pm}$and three Goldstone bosons, the latter three giving mass to the $W^{ \pm}$and $Z$ gauge bosons. This potential has in principle 8 independent parameters. However, because $v$ is fixed by, e.g., the $W^{ \pm}$ boson mass, only 7 independent parameters remain to be chosen, which we take to be $m_{h}, m_{H}, m_{A}$, $m_{H^{ \pm}}, \tan \beta=v_{2} / v_{1}, \alpha$ and $M^{2}$. The angle $\beta$ is the rotation angle from the group eigenstates to the mass eigenstates in the CP-odd and charged Higgs sector. The angle $\alpha$ is the corresponding rotation angle for the CP-even Higgs sector. The parameter $M^{2}$ is defined as $M^{2}=m_{3}^{2} /(\sin \beta \cos \beta)$ and is a measure of how softly the discrete symmetry is broken. The definition of $\alpha$ and the relation among physical scalar masses and coupling constants are shown in Ref. [9] for definiteness.

The discrete symmetry imposed on the potential, when extended to the Yukawa Lagrangian, guarantees that FCNCs are not present at tree level, as fermions of a given electric charge couple to no more than one Higgs doublet [10]. There are a total of four possible combinations [4] and therefore four variations of the basic model. Writing the most general Yukawa interaction under the $Z_{2}$ symmetry as

$$
\mathcal{L}_{\text {Yukawa }}^{\text {2HDM }}=-\bar{Q}_{L} Y_{u} \widetilde{\Phi}_{u} u_{R}-\bar{Q}_{L} Y_{d} \Phi_{d} d_{R}-\bar{L}_{L} Y_{\ell} \Phi_{\ell} \ell_{R}+\text { h.c. },
$$


where $\Phi_{f}(f=u, d$ or $\ell)$ is either $\Phi_{1}$ or $\Phi_{2}$, the four independent $Z_{2}$ charge assignments on quarks and charged leptons can be summarised in table \ [4, 11, 12]. We define as Type I the model where only the

\begin{tabular}{|c||c|c|c|c|c|c|}
\hline & $\Phi_{1}$ & $\Phi_{2}$ & $u_{R}$ & $d_{R}$ & $\ell_{R}$ & $Q_{L}, L_{L}$ \\
\hline Type I & + & - & - & - & - & + \\
Type II & + & - & - & + & + & + \\
Type X & + & - & - & - & + & + \\
Type Y & + & - & - & + & - & + \\
\hline
\end{tabular}

TABLE I: Variation in charge assignments of the $Z_{2}$ symmetry defining the 2HDM Types discussed in this work.

doublet $\Phi_{2}$ couples to all fermions; Type II, the one similar to the Minimal Supersymmetric Standard Model (MSSM), is the model where $\Phi_{2}$ couples to up-type quarks and $\Phi_{1}$ couples to down-type quarks and leptons; a Type Y [5] (or Type III) model is built such that $\Phi_{2}$ couples to up-type quarks and to leptons and $\Phi_{1}$ couples to down-type quarks and finally in a Type X [5] (or Type IV model) $\Phi_{2}$ couples to all quarks and $\Phi_{1}$ couples to all leptons.

We will now discuss the constraints on these 2HDM Types and in particular the existence of a light charged Higgs boson in this context. The parameter space of the aforementioned 2HDMs (or 2HDM Types), as well as of the family of models with similar phenomenology, is limited by experimental data and theoretical constraints (such as vacuum stability and perturbative unitarity). It is well known that in the 2HDM Type II a charged Higgs with a mass below $\approx 300 \mathrm{GeV}$ is disallowed by the constraints from the measurement of $b \rightarrow s \gamma[\underline{6}$. The same bound applies to model Type Y. However, because in models Type I and $\mathrm{X}$ the same doublet couples to both up- and down-type quarks, the bound can be relaxed to $100 \mathrm{GeV}$ or even less depending on the value of $\tan \beta$. In this work we are interested in the light charged Higgs boson mass region, that is, the one below the $m_{t}+m_{b}$ threshold. Hence, only models Type I and $\mathrm{X}$ from a CP-conserving 2HDM (and models that contain them as submodels [8]) allow for the existence of a light charged Higgs state. Finally, we note that we are not considering models that due to a larger particle content, like for instance the MSSM, have the bound on the charged Higgs boson mass relaxed in definite regions of the parameter space as a consequence of extra contributions to processes like $b \rightarrow s \gamma$.

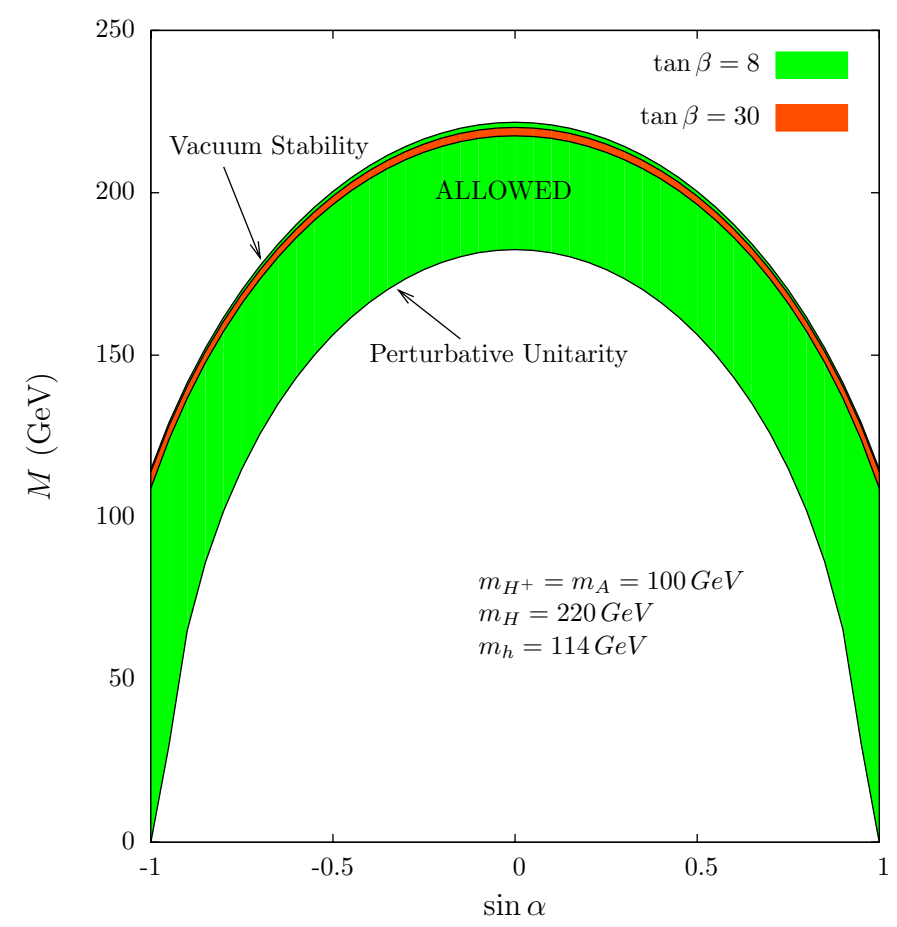

FIG. 1: Region of parameter space allowed in the plane $\left(M^{2}, \sin \alpha\right)$ when vacuum stability and perturbative unitarity constraints are considered. This plot is valid for all Yukawa versions of the basic 2HDM prior to enforcing the experimental constraints. 
There are other bounds that constrain the $2 \mathrm{HDMs}$ which deserve a brief comment. New contributions to the $\rho$ parameter stemming from Higgs states [13] have to comply with the current limits from precision measurements [14]: $|\delta \rho| \lesssim 10^{-3}$ - there are limiting cases though, related to an underlying custodial symmetry, where the extra contributions to $\delta \rho$ vanish. These limits are $m_{h} \simeq m_{H^{ \pm}}$and $\sin (\beta-\alpha) \simeq 0$, $m_{H} \simeq m_{H^{ \pm}}$and $\sin (\beta-\alpha) \simeq 1$, and finally $m_{H^{ \pm}} \simeq m_{A}$. Values of $\tan \beta \lesssim 1$ together with a charged Higgs boson with a mass below a value of the order $100 \mathrm{GeV}$ are disallowed by both the constraints coming from $R_{b}$ and from $B_{q} \bar{B}_{q}$ mixing [15] for all Yukawa versions (also from the $b \rightarrow s \gamma$ [6] measurement already discussed). In models Type II and Y, the branching ratio of the process $B^{+} \rightarrow \tau^{+} \nu$ is enhanced relative to the SM. This is due to a $\tan ^{2} \beta$ factor, with origin in the Yukawa couplings, that is not present in the cases of models Type I and X [2]. Therefore, no relevant bounds can be derived with this process for models Type I and X. Finally, the constraints that would arise from the precise measurements of the muon anomalous magnetic moment $(g-2)_{\mu}$ are irrelevant due to the large values of the masses involved in the process [16].

The theoretical bounds related to perturbative unitarity [17] and vacuum stability [18] (boundness from below) are also imposed and will prove to be very important in defining our benchmark scenarios. The most striking feature is that, as $\tan \beta$ grows, the allowed values of $M$ shrink to a tiny region that depends mainly on $m_{H}$ and $\sin \alpha$. This is extremely important because, as we will see later, when looking for the parameter space where a large enhancement of resonant cross sections is possible, one should be careful to definitely be in the parameter space region where perturbative unitarity and vacuum stability are respected. In figure 1 we present the allowed region for the $2 \mathrm{HDM}$ parameter space when theoretical constraints, vacuum stability and perturbative unitarity, are taken into account, in the plane $\left(M^{2}, \sin \alpha\right)$. We have chosen $\tan \beta=8$ and $\tan \beta=30$ and values for the masses allowed by all other experimental constraints. It is clear that, as $\tan \beta$ grows, the allowed parameter region shrinks. Inside this region of the parameter space, we can look for the largest possible enhancement of the cross sections. Finally, after choosing a CP-conserving minimum, the $2 \mathrm{HDM}$ vacuum is naturally protected against charge and $\mathrm{CP}$ breaking [19]. There are no other bounds relevant for our analysis.

\section{III. $H^{ \pm}$PRODUCTION AND DECAY CHANNELS AT HADRON COLLIDERS IN 2HDM}

This section describes all relevant production and decay channels of charged Higgs bosons within 2HDMs at the LHC. Since a charged Higgs boson couples to fermions and bosons proportionally to their masses, it will predominantly be produced in connection with $\tau^{\prime}$ 's, $b$ 's and $t^{\prime}$ 's as well as $W^{ \pm}$'s and $Z$ 's or indeed in association with other scalars. Besides, it will also decay into these states. As intimated, the discussion here will be focused on a light charged Higgs boson, that is, below the so-called threshold or transition region, when $m_{H^{ \pm}} \approx m_{t}$. We will start by listing the most significant production modes which will then be discussed in detail in the following subsections. We can have single $H^{ \pm}$production [20 29]:

$$
\begin{aligned}
g g, q \bar{q} & \rightarrow b \bar{t} H^{+} \text {and } \bar{b} t H^{-}, \\
b Q & \rightarrow b Q^{\prime} H^{+} \text {and } b Q^{\prime} H^{-}, \\
c s & \rightarrow H^{ \pm}(+j e t), \\
g g, b \bar{b} & \rightarrow W^{-} H^{+} \text {and } W^{+} H^{-}, \\
q Q & \rightarrow S H^{+} \text {and } S H^{-},
\end{aligned}
$$

where $S$ is a neutral scalar $(S=h, H, A)$, or pair production [30 33] (see also [34]):

$$
\begin{aligned}
g g, b \bar{b} & \rightarrow H^{+} H^{-}, \\
q \bar{q} & \rightarrow H^{+} H^{-}, \\
q Q & \rightarrow q^{\prime} Q^{\prime} H^{+} H^{-},
\end{aligned}
$$

where $q, q^{\prime}, Q, Q^{\prime}$ represent (anti)quarks (other than $b$ 's and $t$ 's). Notice that process (3a) contains as subprocesses both top-antitop production followed by the decay $\bar{t} \rightarrow \bar{b} H^{-}$(and c.c.) and $b g \rightarrow t H^{-}$ (and c.c.): see, e.g., [35]. The process $q Q \rightarrow S H^{+}$and $S H^{-}$requires a more elaborate analysis because the decays of the scalar that is produced together with the charged Higgs boson is Yukawa Type model dependent [36, 37]. A complete study of this process is in progress and will be presented elsewhere [38]. 
Although the charged Higgs boson has a number of possible decay modes, in models Type I and X and variations thereof, a light charged Higgs boson decays mainly via $H^{+} \rightarrow \tau^{+} \nu$ and $H^{+} \rightarrow c \bar{s}$. The BR of each channel depends exclusively on the charged Higgs boson mass and on $\tan \beta$ as long as the decays to neutral scalars and a $W^{ \pm}$boson are kinematically forbidden. It is well known that a very light (neutral) CP-even Higgs state is still allowed in the context of multi-Higgs doublets. In 2HDMs, it suffices to take a very small value of $\sin (\beta-\alpha)[39$ ] to avoid the LEP SM Higgs bound [40]. In this work we will consider the mass of the lightest CP-even Higgs boson to be above $100 \mathrm{GeV}$, disallowing therefore the decay $\mathrm{H}^{+} \rightarrow W^{+} h$. The mass of the remaining neutral scalars are also considered to be above $100 \mathrm{GeV}$. For each production process discussed, we will briefly comment on the very light CP-even Higgs scenario, if pertinent.
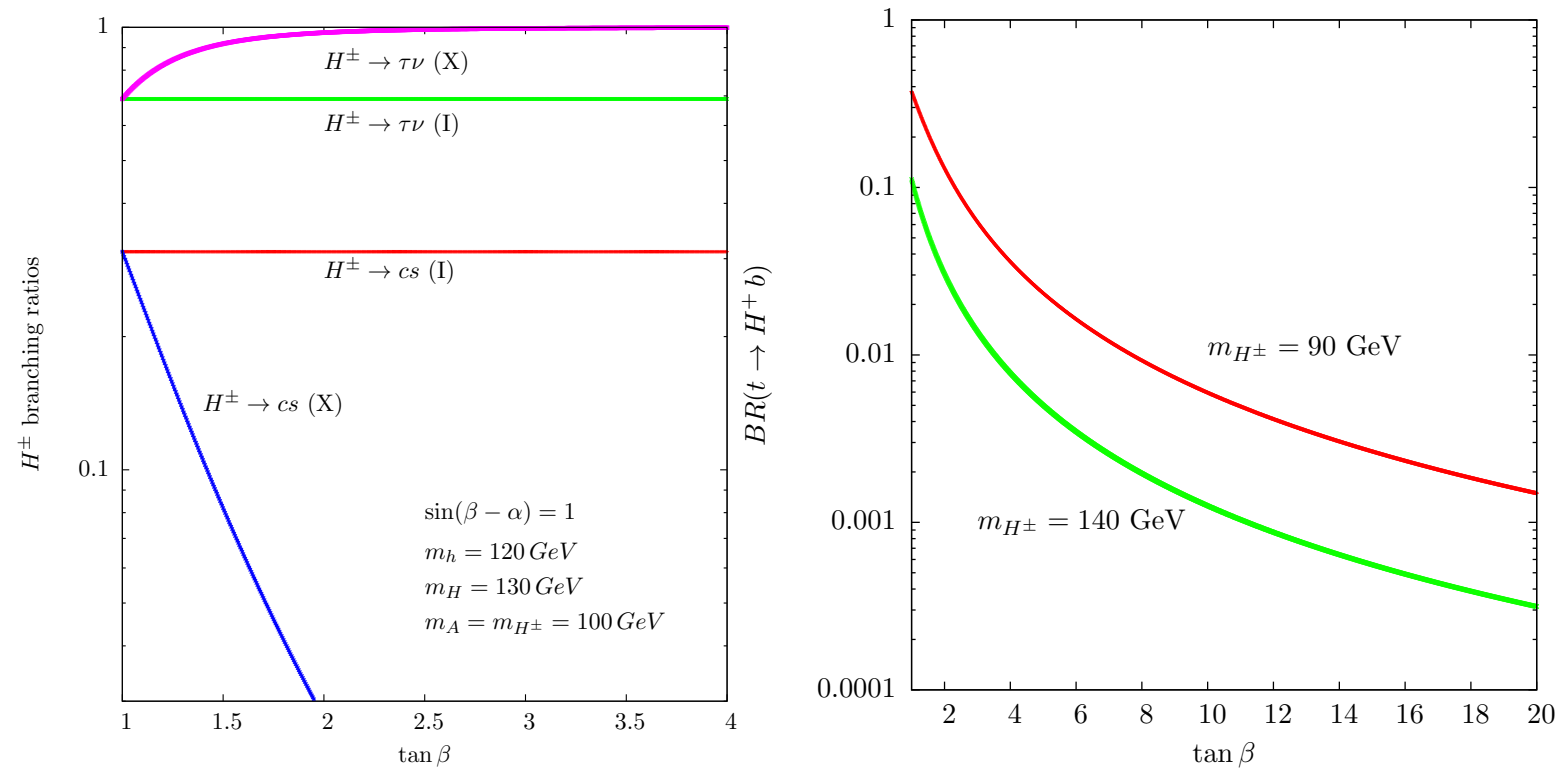

FIG. 2: In the left panel we present the charged Higgs BRs for $m_{H^{ \pm}}=100 \mathrm{GeV}$ as a function of $\tan \beta$ in models Type I and X. In the right panel we show $\operatorname{BR}\left(t \rightarrow H^{+} b\right)$ as a function of $\tan \beta$ for two values of the charged Higgs boson mass.

In the left panel of figure 2 we present the BRs for $m_{H^{ \pm}}=100 \mathrm{GeV}$ as a function of $\tan \beta$ in models Type I and $\mathrm{X}$ (the values of the remaining parameters are shown in the figure). It is clear that, in this type of models, $H^{+} \rightarrow \tau^{+} \nu$ is by far the dominant decay mode. Because the charged Higgs boson width depends only on $\tan \beta$ and on the charged Higgs boson mass, the plot is representative of all $m_{H^{ \pm}}$'s values below the $t \bar{b}$ threshold provided no light neutral scalars are present. At the LHC, the most promising search mode for a light charged Higgs boson is $p p \rightarrow \bar{t} t$ with subsequent decays to $\bar{b} b W^{ \pm} H^{\mp}$. Therefore, to understand how the (anti)top decays change relative to the SM, where the $\operatorname{BR}\left(t \rightarrow W^{+} b\right)$ is close to $100 \%$, we show in the right panel of the figure the $\operatorname{BR}\left(t \rightarrow H^{+} b\right)$ as a function of $\tan \beta$ for two values of the charged Higgs boson mass. Contrary to the case of the MSSM and MSSM-like versions of a Type II $2 \mathrm{HDM}$, this $\mathrm{BR}$ falls very rapidly with $\tan \beta$ here, which makes this mode useless for values of $\tan \beta \gtrsim 10$ and even more so as the charged Higgs boson mass approaches the top quark one. Therefore, we conclude that in these models light charged Higgs bosons decay predominantly to $\tau \nu$ and that searches based on (anti)top-quark decays to charged Higgs bosons are only effective for low to moderate values of $\tan \beta$. We will now discuss each production mode in turn.

\section{A. Double top production}

The most promising searches for a light charged Higgs at the LHC were performed in the $p p \rightarrow t \bar{t} \rightarrow$ $H^{ \pm} b W^{ \pm} \bar{b} \rightarrow \tau \nu b \bar{b} q \bar{q}$ channel. This is the first process on our list (3a) where both top and anti-top are produced on-shell. This production mode depends only on $\tan \beta$ and on the charged Higgs mass via both the top and the charged Higgs boson BRs. Therefore, the region of parameter space probed can be shown in the $\left(\tan \beta, m_{H^{ \pm}}\right)$plane and is independent of all other 2HDM parameters. Both the ATLAS [41] and 

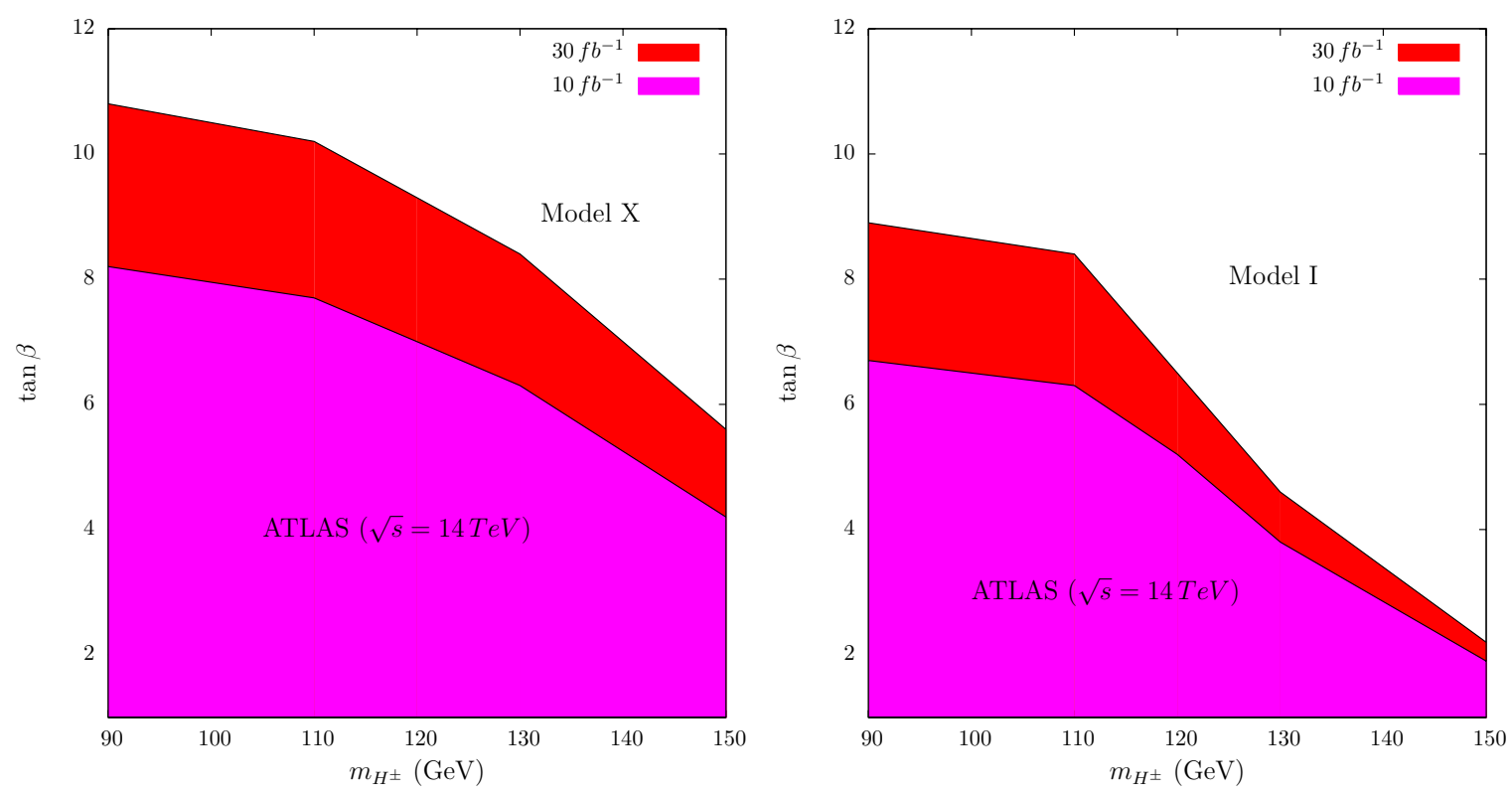

FIG. 3: Region of the parameter space excluded by the ATLAS collaboration for $\sqrt{s}=14 \mathrm{TeV}$ in models Type $\mathrm{X}$ (left) and Type I (right) using the channel $p p \rightarrow t \bar{t} \rightarrow H^{ \pm} b W^{ \pm} \bar{b} \rightarrow \tau \nu b \bar{b} q \bar{q}$. The plot was done using the data in [41].

the CMS [42] collaborations have performed full detector analyses in this channel and have used them to explore the parameter space of the MSSM. We can now use their raw results to constrain the parameter space of $2 \mathrm{HDMs}$ Type I and X. In figure 3 we present the region of the parameter space that can be excluded at 95\% CL in model Type X (left panel) and Type I (right panel) at the LHC after collecting 10 and $30 \mathrm{fb}^{-1}$ of integrated luminosity using the results from the ATLAS collaboration 41]. Very briefly, the procedure to extract the data from [41] was the following. The ATLAS collaboration has generated the signal $p p \rightarrow t \bar{t} \rightarrow H^{ \pm} b W^{ \pm} \bar{b} \rightarrow \tau \nu b \bar{b} q \bar{q}$ using MSSM tools that are only relevant in the calculation of $B R\left(t \rightarrow H^{ \pm} b\right)$ and $B R\left(H^{ \pm} \rightarrow \tau \nu\right)$ - all the remaining cross sections and branching ratios are SM like (including all the backgrounds). We then took the final values in [41] for a given point in parameter space, divided them by the MSSM values for $B R\left(t \rightarrow H^{ \pm} b\right)$ and $B R\left(H^{ \pm} \rightarrow \tau \nu\right)$ and multiply them for the corresponding values for the $2 \mathrm{HDM}$ model under study.

Contrary to the MSSM scenario described by the ATLAS collaboration in [41], the 2HDM parameter space will not be completely covered with a luminosity of $30 \mathrm{fb}^{-1}$. As previously discussed, $2 \mathrm{HDMs}$ Type I and X have Yukawa couplings proportional to $1 / \tan \beta$. Hence, contrary to the MSSM where a term $m_{b} \tan \beta$ (where $m_{b}$ is the bottom-quark mass) gives large contributions for large $\tan \beta$, in Types I and X this term in now $m_{b} / \tan \beta$, thus it decreases with $\tan \beta$. Moreover, an increase in luminosity will make the excluded region to grow to larger values of $\tan \beta$, but because $\operatorname{BR}\left(t \rightarrow \bar{b} H^{+}\right)$always decreases as $\tan \beta$ grows, very large values of $\tan \beta$ will never be probed with this process.

\section{B. Single top production}

In this section we estimate the contribution of the process $p p \rightarrow H^{ \pm} b j$ with $H^{ \pm} \rightarrow \tau \nu_{\tau}$ to light charged Higgs boson searches at the LHC in 2HDMs. This mode consists mainly of $t$-channel plus $s$-channel single top production followed by the decay $t \rightarrow H^{+} \bar{b}$. Our goal is to understand whether this process, that again only depends on the charged Higgs boson mass and $\tan \beta$, could contribute to improve the region already scrutinised by $p p \rightarrow t \bar{t} \rightarrow H^{ \pm} b W^{ \pm} \bar{b} \rightarrow \tau \nu b \bar{b} q \bar{q}$ presented in the previous section. A first study for this process was presented in [21]. To estimate single top contribution, we take as our signal the process

$$
p p \rightarrow H^{ \pm} b j \rightarrow b j \tau \nu_{\tau} .
$$


Regarding the background, we consider the irreducible background process

$$
p p \rightarrow \operatorname{bjl}(\tau, \mu, e) \nu_{\tau, \mu, e}
$$

and the reducible one

$$
p p \rightarrow t \bar{t} \rightarrow W^{+} b W^{-} \bar{b}
$$

where both $W^{ \pm}$'s can decay semi-leptonically, fully leptonically or fully hadronically. The details of the parton level analysis are presented in Appendix A. For the signal, we have varied the charged Higgs boson mass from $m_{H^{ \pm}}=100 \mathrm{GeV}$ to $m_{H^{ \pm}}=140 \mathrm{GeV}$ in $10 \mathrm{GeV}$ steps and the results are for $\tan \beta=1.5$. The signal was generated with CalcHEP [43] and so was the $t \bar{t}$ background. The single top background, $p p \rightarrow \operatorname{bjl}(\tau, \mu, e) \nu_{\tau, \mu, e}$, was instead generated with MadGraph/MadEvent [44]. The partonic CM energy

\begin{tabular}{|c|c|c|c|c|c|}
\hline & $m_{H^{ \pm}}=100 \mathrm{GeV}$ & $m_{H^{ \pm}}=110 \mathrm{GeV}$ & $m_{H^{ \pm}}=120 \mathrm{GeV}$ & $m_{H^{ \pm}}=130 \mathrm{GeV}$ & $m_{H^{ \pm}}=140 \mathrm{GeV}$ \\
\hline \multicolumn{6}{|l|}{ Process } \\
\hline Signal & $379.4 f b$ & $274.4 f b$ & $202.7 f b$ & $118.9 \mathrm{fb}$ & $65.5 \mathrm{fb}$ \\
\hline $\mathrm{Bg}$ (single top) & $1705.4 \mathrm{fb}$ & & & & \\
\hline $\mathrm{Bg}(t \bar{t}$ semi-leptonic $)$ & $683.1 \mathrm{fb}$ & & & & \\
\hline $\mathrm{Bg}(t \bar{t}$ leptonic $)$ & $393.6 \mathrm{fb}$ & & & & \\
\hline$\sigma_{S} / \sigma_{B}$ & 0.14 & 0.098 & 0.073 & 0.042 & 0.023 \\
\hline$\sigma_{S} / \sqrt{\sigma_{B}}\left(f b^{1 / 2}\right)$ & 7.19 & 5.20 & 3.84 & 2.25 & 1.24 \\
\hline
\end{tabular}
was chosen as both the renormalisation and factorisation scale.

TABLE II: Cross section for the signal $p p \rightarrow H^{ \pm} b j \rightarrow b j \tau \nu_{\tau}$ and for the irreducible $\left(p p \rightarrow b j l(\tau, \mu, e) \nu_{\tau, \mu, e}\right)$ and main reducible $\left(p p \rightarrow t \bar{t} \rightarrow W^{+} b W^{-} \bar{b}\right)$ backgrounds after all cuts have been applied. The analysis is performed for model Type $\mathrm{X}$ with $\tan \beta=1.5$ and the single top process has a negligible dependence on the remaining parameters of the 2HDM. (Background rates are independent of $m_{H^{ \pm}}$as the latter parameter is not used in the selection, see Appendix A.)

By performing the analysis presented in Appendix A we have found the results presented in table [I] Our results show that, for a mass of $100 \mathrm{GeV}$, an exclusion of $\tan \beta \lesssim 5$ could be expected at $95 \%$ CL for a collected luminosity of $10 \mathrm{fb}^{-1}$ and $\tan \beta \lesssim 7$ for $30 \mathrm{fb}^{-1}$, for model Type X. The results then degrade rapidly with growing charged Higgs boson mass as compared to the previous process of $t \bar{t}$ production, e.g, for a $140 \mathrm{GeV} H^{ \pm}$mass, even a value of $\tan \beta=3$ is not reachable with $30 \mathrm{fb}^{-1}$ of integrated luminosity at $95 \%$ CL. As discussed in Appendix A, these results will most probably prove to be optimistic as this is a parton level analysis. However, we believe to have made a case for the need of further studies of this process and we do think that a full detector level analysis would be worth performing. Finally, in accordance with the previous section, the results are slightly worse for model Type I due to the reduction of the decay rate to $\tau \nu$ when compared to model Type $\mathrm{X}$.

\section{Direct charged Higgs boson production}

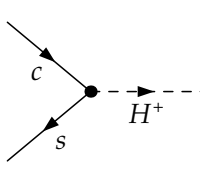

(a)

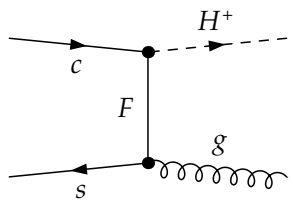

(b)

FIG. 4: Representative Feynman diagrams for direct charged Higgs boson production and charged Higgs boson plus jet production. (Herein, $F$ represents a fermion, as appropriate.)

The last process on our list that depends only on the charged Higgs boson mass and on tan $\beta$ is direct charged Higgs boson production $c s \rightarrow H^{ \pm}(+$jet$)$and is shown in figure 4 . As discussed before, contrary 
to the MSSM and 2HDMs Type II and Y, in models Type I and X, the couplings of the charged Higgs to both up- and down-type quarks is proportional to $1 / \tan \beta$. This is the reason why we write as initial state only $c s$ and not $c b+c s$ - the $c b$ initiated mode can be the dominant one for direct production in the above models, as discussed in 22, 23. In models Type I and X the ratio between the $c b$ and the $c s$ initiated contributions is of the order $V_{c b}^{2}\left(m_{c}^{2}+m_{b}^{2}\right) / m_{c}^{2}$ which makes the $c b$ contribution negligible for all charged Higgs masses and for all $\tan \beta$ values.

A detailed experimental study for direct charged Higgs boson production with subsequent decay to $\tau \nu$ was performed by CMS 24]. In that study, it was shown that values of $\tan \beta$ above 15 could be excluded at $99 \% \mathrm{CL}$ for $m_{H^{ \pm}}=200 \mathrm{GeV}$ for the MSSM. According to [23], this corresponds to a cross section of the order of a few hundreds of $f b$. However, for models Type I and X, the cross sections are below 1 $f b$ even for $\tan \beta=1$, where they reach their maximum value. If we take the lower limit for the mass, $m_{H^{ \pm}}=100 \mathrm{GeV}$, then the typical cross section is of the order of $700 \mathrm{fb}$ for $\tan \beta=1$. This seems like a large value but there are two drawbacks. First, the value $m_{H^{ \pm}}=100 \mathrm{GeV}$ is close to the $W^{ \pm}$mass and therefore the irreducible background will make the detection much harder. Second, as soon as we move to, say, $\tan \beta=4$ the cross section is reduced to values of the order of $50 \mathrm{fb}$. Hence, we conclude that this process will not give any significant contribution to the limits in the $\left(m_{H^{ \pm}}, \tan \beta\right)$ plane established in the previous sections for 2HDM Type I and X.

\section{Charged Higgs boson pair production}

Charged Higgs pair production in 2HDMs at the LHC proceeds via three different channels, $g g \rightarrow H^{+} H^{-}$, $b \bar{b} \rightarrow H^{+} H^{-}$and the Drell-Yan (DY) process $q \bar{q} \rightarrow H^{+} H^{-}$, where $q$ stands for a light quark. The first two processes are presented in figure 5 whilst the last one is shown in figure 6. The most recent study that compares all three processes in the MSSM was performed in [31]. Therein, it was shown that, for the MSSM, the main contribution comes from DY except for very large values of $\tan \beta$ (above 50) where gluon fusion gives the main contribution. However, when we consider 2HDMs of Type I and X, there is never a $\tan \beta$ enhancement related to the Yukawa couplings of charged Higgs bosons to quarks as these are always proportional to $1 / \tan \beta$. Conversely, because in $2 \mathrm{HDMs}$ we are not constrained by relations between the scalar masses, large cross sections can be obtained if we consider resonant production of neutral Higgs bosons decaying into pairs of charged ones. In this scenario, cross sections of $p b$ order can be reached if one considers a further enhancement due to Higgs self-couplings. In this scenario, the $b \bar{b} \rightarrow H^{+} H^{-}$mode (diagrams (a) and (b) in figure 5) is always much smaller than the gluon fusion mode (diagrams (c)-(f) in figure 5), especially for large $\tan \beta$, because the Yukawa enhancement only happens in 2HDM Type II, Type Y and in the MSSM. As it has been noted that the large enhancement to the production cross section can only happen in resonant production, it is always diagram (d) (and partly (a)) in figure 5 that dominates in these circumstances, through the exchange of a heavy CP-even Higgs.

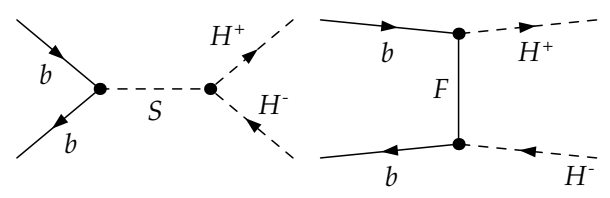

(a)

(b)

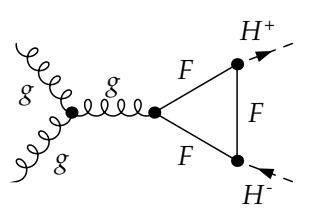

(c)

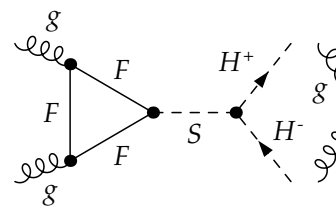

(d)

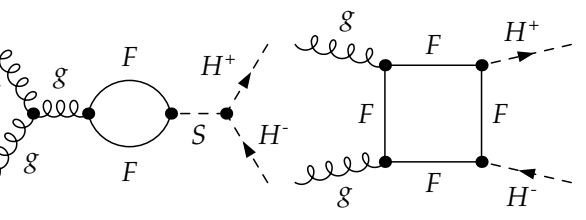

(e) (f)

FIG. 5: Representative Feynman diagrams for charged Higgs boson pair production via $b \bar{b},(\mathrm{a})$-(b), and $g g$, (c)-(f), fusion. (Herein, $F$ represents a fermion, as appropriate, whereas $S$ a neutral Higgs boson, as appropriate.) 


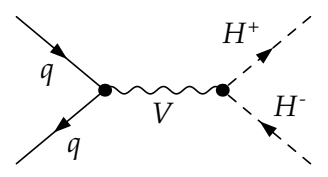

FIG. 6: Feynman diagrams for DY charged Higgs boson pair production. (Herein, $V$ represents a neutral gauge boson, as appropriate.)

We showed in previous sections that, for a charged Higgs boson mass of $100 \mathrm{GeV}$, the processes that depend only on $\tan \beta$ and on the charged Higgs boson mass itself will at least exclude a region where $\tan \beta \lesssim 8$ (e.g., in model Type $\mathrm{X}$ for $10 \mathrm{fb}^{-1}$ of integrated luminosity and $\sqrt{s}=14 \mathrm{TeV}$ ). The situation could even be improved when a thorough study for single top production is combined with the one already performed for the $t \bar{t}$ case by the ATLAS and the CMS collaborations. As the charged Higgs mass grows, the excluded range of $\tan \beta$ shrinks: e.g., for a $150 \mathrm{GeV}$ charged Higgs boson mass we get $\tan \beta \gtrsim 2$ for model Type I and $\tan \beta \gtrsim 4$ for model Type $\mathrm{X}$, when $10 \mathrm{fb}^{-1}$ are collected. Therefore, we want to see whether large values of $\tan \beta$ can give significant cross sections in charged Higgs boson pair production. This would allow us to look into the high $\tan \beta$ region which we already know will never be accessible with the previous reactions ( $3 \mathrm{a}, 3 \mathrm{~b}$ and $3 \mathrm{c}$ ). However, we first have to understand what can be considered a significant cross section. For this purpose we make use of the studies from [45] made in the context of left-right symmetric models and [46] performed in the context of a 2HDM-like model, where three gauge-singlet right-handed Weyl spinors were added to become the right-handed components of the three Dirac neutrinos. Using these studies [45, 46] as a guide, we conclude that a significant cross section is one of the order of $400 \mathrm{fb}$. This means that, using their analysis for our signal one would be able to start probing a significant parameter region with $30 \mathrm{fb}^{-1}$ for $2 \mathrm{HDM}$ Type $\mathrm{X}$ if the production cross section was of the order of $400 \mathrm{fb}$. The details of the analysis are presented in Appendix B.
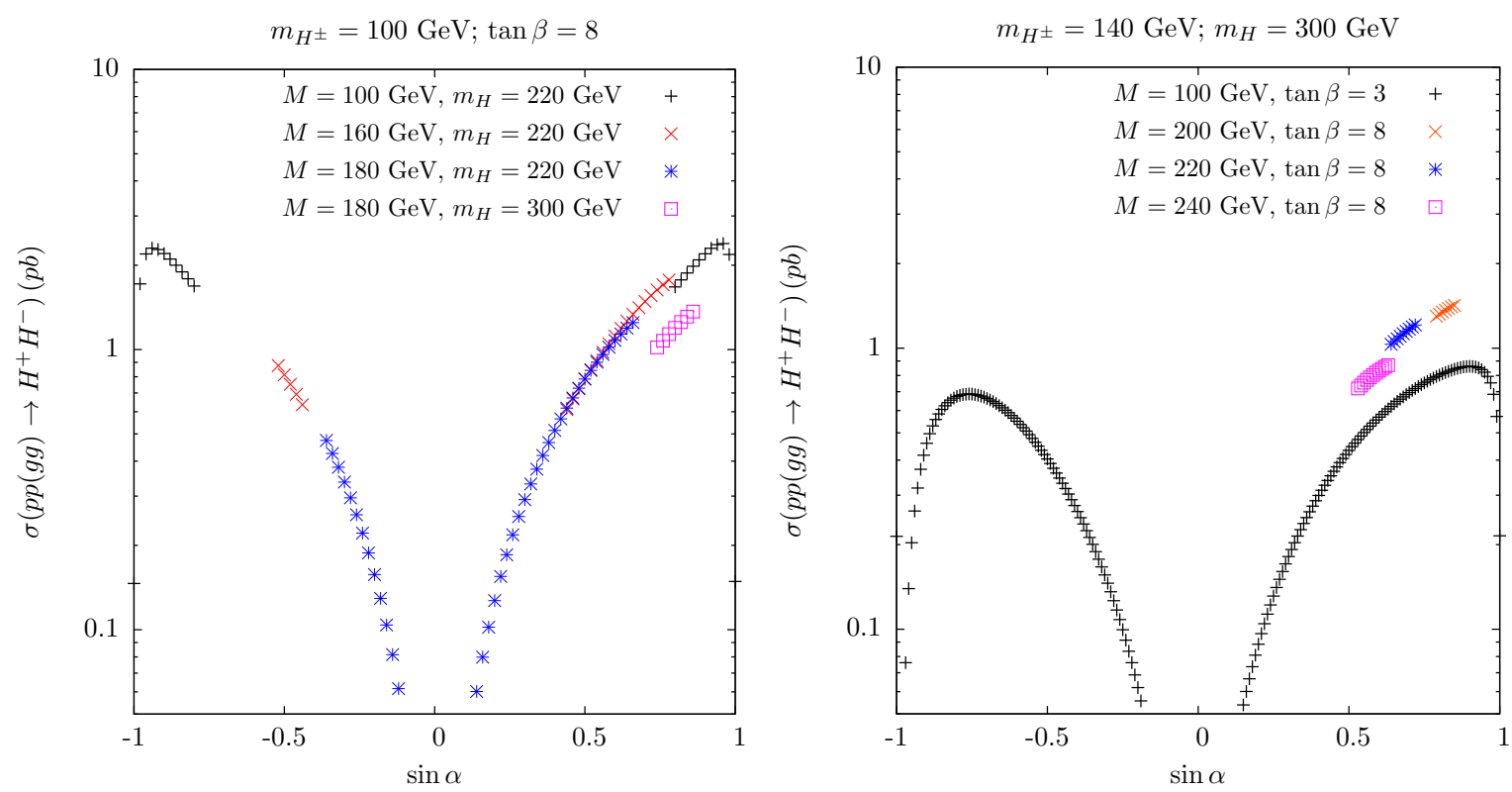

FIG. 7: Production cross section for $p p(g g) \rightarrow H^{+} H^{-}$as a function of $\sin \alpha$ for $m_{H^{ \pm}}=100 \mathrm{GeV}$ (left) and $m_{H^{ \pm}}=140 \mathrm{GeV}$ (right) for a set of chosen values of $\tan \beta, m_{H}$ and $M$.

Before showing our results for charged Higgs pair production, let us first analyse the high $\tan \beta$ behaviour of the cross section. If, in fact, no $\tan \beta$ enhancement can be expected for this process, it will be useless because the previous processes $3 \mathrm{a}, 3 \mathrm{~b}$ and $3 \mathrm{c}$ already constrain a significant region of the $\left(\tan \beta, m_{H^{ \pm}}\right)$ plane. It is easy to show that for high $\tan \beta$ we have

$$
g_{H H^{+} H^{-}} g_{H \bar{t} t} \propto \sin \alpha \cos \alpha \tan \beta\left(m_{H}^{2}-M^{2}\right) \quad(\tan \beta \gg 1)
$$


where $g_{H H^{+} H^{-}}$is the coupling of the heavy CP-even Higgs to a charged Higgs pair while $g_{H \bar{t} t}$ is the $H$ Yukawa coupling to top quarks. Therefore, it becomes clear that the cross section increases with $\tan ^{2} \beta$ and can be used to explore the high $\tan \beta$ region. Asymptotically, there is also a term independent of $\tan \beta$ of the form $\sin ^{2} \alpha\left(2 m_{H^{ \pm}}^{2}-M^{2}\right)$ that can be important for moderate values of $\tan \beta$. It is interesting to note that the lightest $\mathrm{CP}$-even Higgs state has a similar behaviour in the same regime

$$
g_{h H^{+} H^{-}} g_{h \bar{t} t} \propto \sin \alpha \cos \alpha \tan \beta\left(m_{h}^{2}-M^{2}\right) \quad(\tan \beta \gg 1)
$$

where the couplings refer now to the lightest CP-even Higgs state. Hence, if both $H$ and $h$ have masses above two times the charged Higgs boson mass, their contributions will be indistinguishable at high $\tan \beta$. In the left panel of figure 7 we present the cross section for $g g \rightarrow H^{+} H^{-}$with $m_{H^{ \pm}}=100 \mathrm{GeV}$, $\tan \beta=8$ and for several values of $\left(M, m_{H}\right)$ as a function of $\sin \alpha$. Some lines on the plots end or/and start abruptly due to the theoretical constraints: this means that these are constraints that will not change with time and can be regarded as a part of the parameter space that is definitely excluded. It is clear that large cross sections can be obtained for various regions of the parameter space as long as the production is resonant. In the right panel we show the cross section for $m_{H^{ \pm}}=140 \mathrm{GeV}$ and for a fixed $m_{H}$ of $300 \mathrm{GeV}$ for several values of $(M, \tan \beta)$ as a function of $\sin \alpha$. Again, we see that values of the cross section close to $1 p b$ can easily be obtained. The light CP-even Higgs mass was taken to be 120 $\mathrm{GeV}$ while the CP-odd boson mass $m_{A}$ was chosen so as to avoid the $\delta \rho$ constraint. The calculations were performed with the packages FeynArts [47] and FormCalc [48]. The scalar integrals were evaluated with LoopTools [49] and the CTEQ6L parton distribution functions [50] were used. Again, the partonic CM energy was chosen as both the factorisation and renormalisation scale. The value of the DY cross section was not taken into account. This means that taking a charged Higgs mass of $100 \mathrm{GeV}$ a value of the order of $200 \mathrm{fb}$ should be added to the values presented in the plot. We have repeated the calculation for the same processes but initiated by $b \bar{b}$ and found that the cross sections are usually at least one order of magnitude below the $g g$ initiated process.

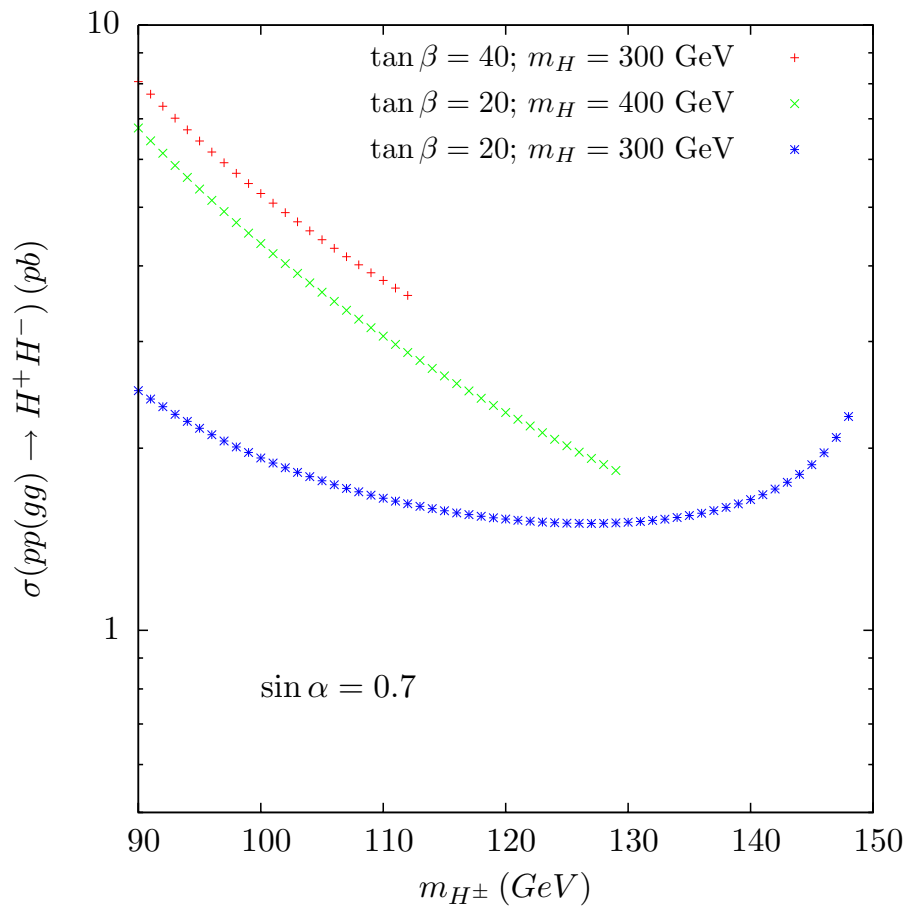

FIG. 8: Production cross section for $p p(g g) \rightarrow H^{+} H^{-}$as a function of the charged Higgs boson mass. The values of $\sin \alpha$ were chosen so as to maximise the cross section while complying with all the constraints.

In figure 8 we present the total cross section for $p p(g g) \rightarrow H^{+} H^{-}$as a function of the charged Higgs boson mass. The values of $\tan \beta$ and $\sin \alpha$ were chosen so as to maximise the cross section while in agreement with all constraints. These are the maximum values the cross section can attain, a few $p b$, which is not surprising because the production cross section via $g g$ fusion for a SM Higgs boson is of the order of a few tenths of $p b$. Even considering the decay into a pair of charged Higgs bosons to be close to 100\%, 
the final cross section values cannot exceed a few $p b$. We note that this process is highly dependent on the available channels for the $H$ state to decay into, though. If, for example, we would close the channel $H \rightarrow h h$ by increasing the lightest CP-even Higgs boson mass, the cross section would become larger in some regions of the parameter space. We should however note the importance of this process in probing the large $\tan \beta$ region. As in these models the charged Higgs Yukawa couplings decrease like $1 / \tan \beta$, only the self-coupling $\mathrm{HH}^{+} \mathrm{H}^{-}$allows for large values of the cross section for high $\tan \beta$. Finally, a word about the very light $\mathrm{CP}$-even Higgs scenario is in order. In the large $\tan \beta$ regime, $\sin (\beta-\alpha) \approx \cos \alpha$. The largest values of the cross section are obtained for $\sin \alpha \approx 0.7$, which means $\sin (\beta-\alpha) \approx \cos \alpha \approx 0.7$. Consequently, the LEP bound forces the lightest CP-even Higgs mass to be above $100 \mathrm{GeV}$. However, we can still get sizeable cross sections for $\sin \alpha$ very close to 1 . In that case, not only a very light CP-even Higgs boson is allowed but, moreover, the decay $H^{+} \rightarrow W^{+} h$ becomes maximal, as it is proportional to $\cos (\beta-\alpha)$.

We end this section with a brief comment on the cross section values for $M^{2}<0$. First, note that the values of $M^{2}$ affect only the processes we define in this work as charged Higgs pair production and Vector Boson Fusion (VBF), via the vertices $H(h) H^{+} H^{-}$. As discussed in [51, 52], negative values of $M^{2}$ can give rise to very large values of the cross section of neutral Higgs pair production as a result of an enhancement of the $H(h) H^{+} H^{-}$self-couplings. The same is true for charged Higgs pair production and VBF. We have previously shown the asymptotic behaviour of the $\mathrm{HH}^{+} \mathrm{H}^{-}$coupling, $g_{H H^{+} H^{-}} g_{H \bar{t} t} \propto \sin \alpha \cos \alpha \tan \beta\left(m_{H}^{2}-M^{2}\right) \quad(\tan \beta \gg 1)$. Therefore, it is clear that the largest values of $g_{H^{+} H^{-}} g_{H \bar{t} t}$ are obtained for $M^{2}<0$. However, we have shown in [53] that, when $M^{2}<0$, $\tan \beta$ is constrained to be small by perturbative unitarity and more so if $\left|M^{2}\right|$ becomes very large. We have shown in [53] that it is very hard to go beyond a value of $\tan \beta \approx 8$ even for $m_{H}=130 \mathrm{GeV}$ - the larger $m_{H}$ is the more constrained $\tan \beta$ is, independently of the value of $M^{2}<0$. Hence, as the range of small to moderate $\tan \beta$ will be covered by the processes that depend only on $\tan \beta$ and on the charged Higgs mass, there is no need for a detailed study of the $M^{2}<0$ scenario.

\section{E. Vector Boson Fusion}

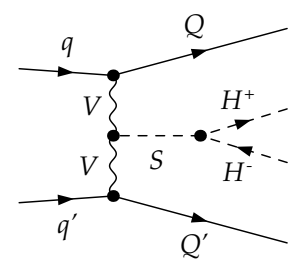

FIG. 9: Feynman diagram for resonant charged Higgs boson production via VBF. (Herein, $S$ represents a neutral Higgs boson, as appropriate.) The complete set of Feynman diagrams for VBF can be found in [33]. However large cross sections are obtained just for resonant production.

In the $\mathrm{SM}, \mathrm{VBF}$ is the closest competitor to Higgs boson production via gluon fusion, in terms of inclusive rates. Although it presents a smaller value for the the total cross section, it has obvious advantages in the analysis due the background reduction accomplished by using the two very forward/backward jets that accompany the Higgs boson in the final state. In the case of $2 \mathrm{HDMs}$ and heavier charged Higgs bosons, VBF can be induced at one-loop level for the case of singly produced $H^{ \pm}$states (see [54, 55] for the case of $e^{+} e^{-}$colliders), however, we have verified here that the corresponding hadro-production rates are negligible.

Production of $H^{ \pm}$pairs is instead possible at tree level from VBF and, like with the previous production process, only the resonant diagram in figure 9 together with an enhanced $\mathrm{HH}^{+} \mathrm{H}^{-}$coupling gives rise to sizeable values of the total cross section. A detailed parton level study for $p p \rightarrow H^{+} H^{-} j j$ was performed for VBF in the context of the MSSM in [33]. This study considered only charged Higgs masses above the $t b$ threshold and therefore the largest number of signal events was obtained for the final state $H^{+} H^{-} j j \rightarrow b \bar{b} W^{+} \tau^{-} \nu j j \rightarrow b \bar{b} j j j j \tau \nu \rightarrow 6 j+\tau+\not p_{T}$. Therein it was shown that in the framework of the MSSM and for the LHC $(\sqrt{s}=14 \mathrm{TeV})$ this final state will be approximately 1000 times below the 


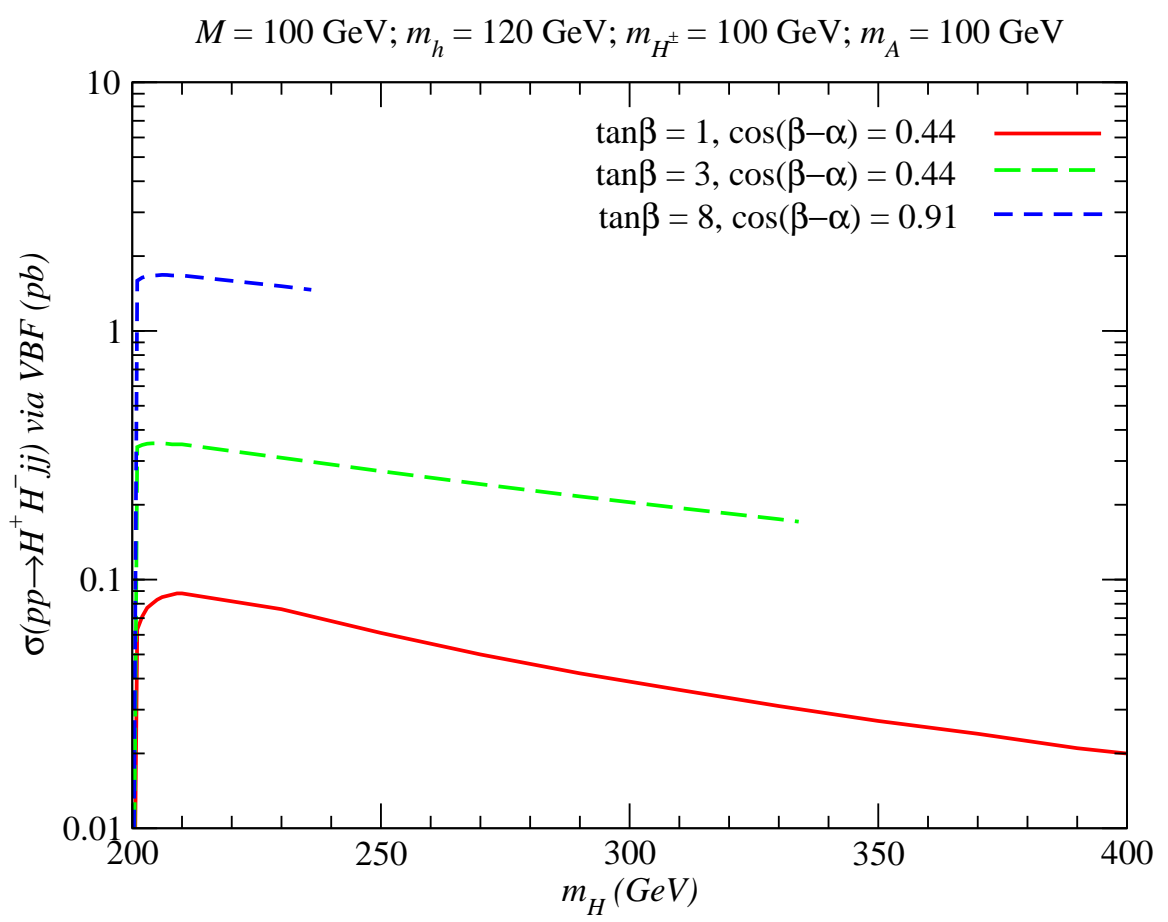

FIG. 10: Production cross section for $p p \rightarrow H^{+} H^{-} j j$ via VBF as a function of the heaviest CP-even Higgs boson mass. The values of $\sin \alpha$ were chosen so as to maximise the cross section while complying with all available constraints.

QCD background. Clearly, below the $t b$ threshold, the largest number of signal events is obtained with final states $H^{+} H^{-} j j \rightarrow 2 \tau+2 j+\not p_{T}$ instead. This is also a cleaner detection mode for which there is no available study in the literature. However, using the SM processes as a guide we would conclude that cross sections close to the $p b$ level will most certainly be accessible at the LHC.

In figure 10 we present the total cross section for $p p \rightarrow H^{+} H^{-} j j$ via VBF as a function of the charged Higgs boson mass. For a given $\tan \beta$, the values of $\sin \alpha$ were chosen so as to maximise the cross section while complying with all the constraints. As we saw before for the process $g g \rightarrow H^{+} H^{-}$, here the lines also end abruptly due to the constraints imposed on the model. Also, like for $g g \rightarrow H^{+} H^{-}$, the largest cross sections are obtained for large $\tan \beta$ which for $\tan \beta=8$ are already of the order of the $p b$. In fact, it is clear from the structure of the couplings that VBF and gluon fusion have exactly the same behaviour with $\sin \alpha$ and $\tan \beta$ for large $\tan \beta$. The $H W^{+} W^{-}$coupling (appearing in VBF) is proportional to $\cos (\beta-\alpha)$ while the $H \bar{t} t$ one is proportional to $\sin \alpha / \sin \beta$ in the models under study (notice that for large $\tan \beta, \cos (\beta-\alpha) \approx \sin \alpha / \sin \beta$ ). This behaviour is shown in figure 11 where we compare the product of the couplings $\left|g_{H_{H^{+} H^{-}}} g_{H t \bar{t}}\right|$, the main contribution in the gluon fusion process, and $\left|g_{H^{+} H^{-}} g_{H W^{+} W^{-}}\right|$, the main contribution in the VBF process (no constraints were applied). It is clear that, as $\tan \beta$ grows, the two processes become indistinguishable from the point of view of the $2 \mathrm{HDM}$ parameter space. Hence, if there is complementarity between the two processes this is only true for small $\tan \beta$. If a light charged Higgs boson is not found, then the small values of $\tan \beta$ will be excluded by $p p \rightarrow t \bar{t} \rightarrow b \bar{b} W^{ \pm} H^{ \pm}$as discussed in section 【IIA In this case, the region of the parameter space probed by $\mathrm{VBF}$ and gluon fusion will be almost the same.

\section{F. Associated production with a $W^{ \pm}$}

Contrary to charged Higgs boson pair production, discussed in the two previous subsections, the process $p p \rightarrow H^{+} W^{-}+H^{-} W^{+}$, as shown in figure 12, does not depend on the Higgs self-couplings. Therefore, 

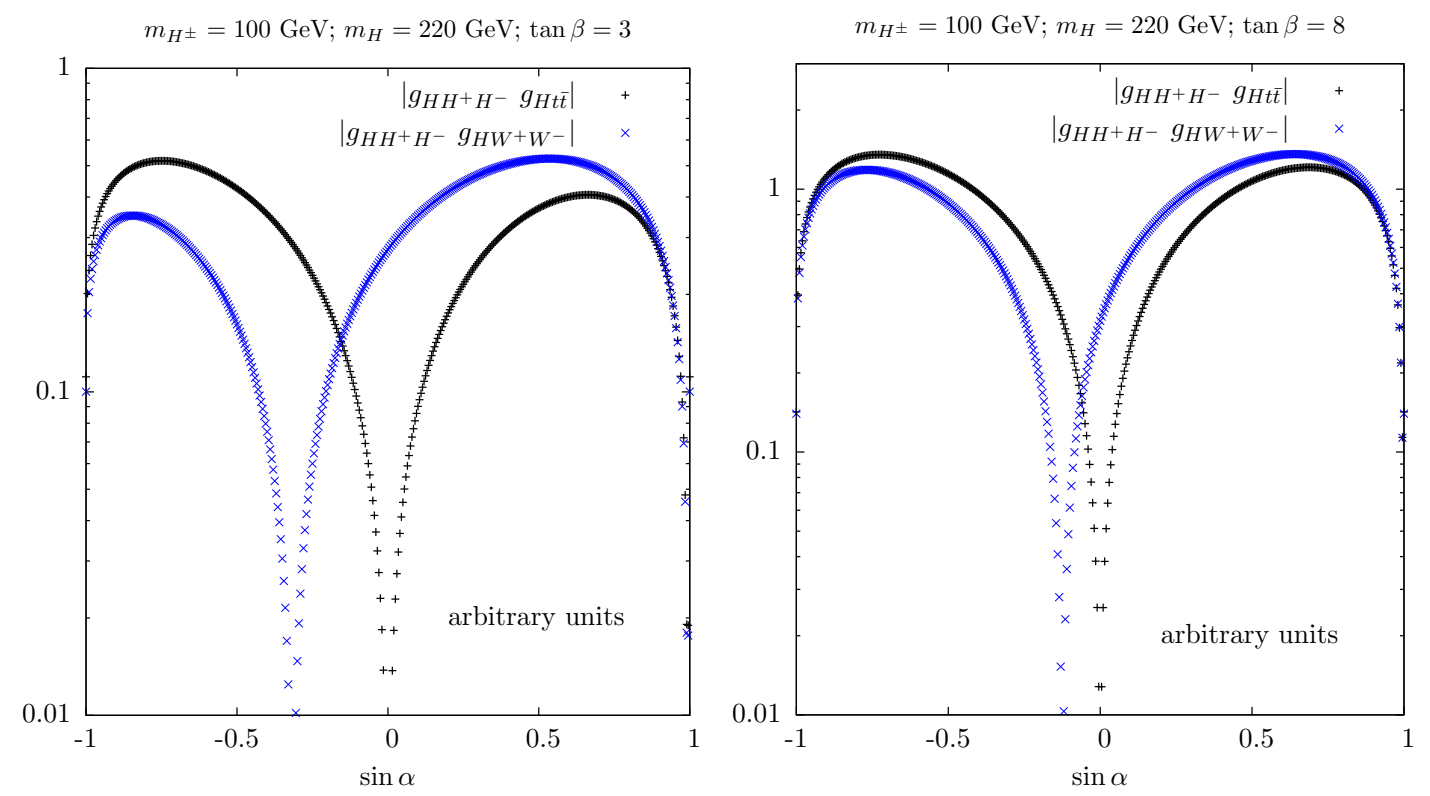

FIG. 11: Comparison between the product of the couplings $\left|g_{H H^{+} H^{-}} g_{H t \bar{t}}\right|$, the main contribution in the gluon fusion process, and $\left|g_{H_{H^{+} H^{-}}} g_{H_{W}+W^{-}}\right|$, the main contribution in the VBF process, for the values of the masses presented in the plot and $\tan \beta=3$ (left) and $\tan \beta=8$ (right). We have chosen $M=170 \mathrm{GeV}$ and the values of $m_{A}$ and $m_{h}$ are irrelevant because the vertices do not depend on those masses.

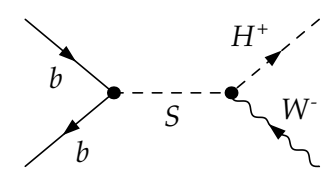

(a)

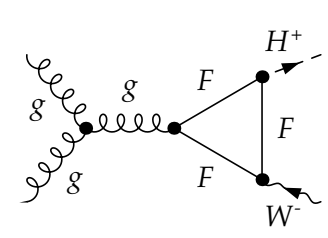

(b)

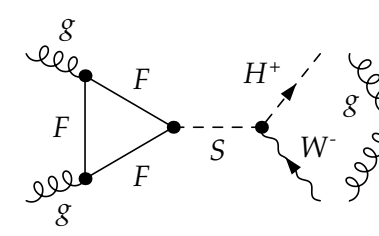

(c)

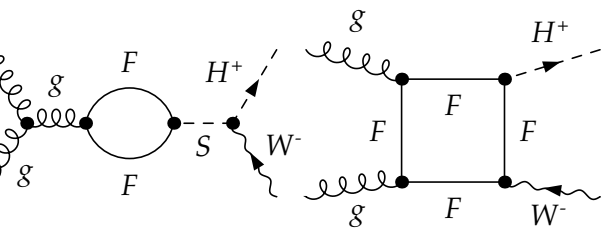

(d)

(e)

FIG. 12: Representative Feynman diagrams for $W^{ \pm} H^{\mp}$ production via $b \bar{b},(\mathrm{a})$, and $g g$, (b)-(e), fusion. (Herein, $F$ represents a fermion, as appropriate, whereas $S$ a neutral Higgs boson, as appropriate.)

it is not suitable to explore the high $\tan \beta$ regime. The reason is simple: gauge couplings, for large $\tan \beta$, are either proportional to $\sin \alpha$ or $\cos \alpha$. As an example, in the limit of very high $\tan \beta$, we have $\left|g_{H W^{+} W_{-}}\right| \approx\left|\sin \alpha g_{H W^{+} W_{-}}^{S M}\right|$ and thus the 2HDM coupling is always smaller than the corresponding SM coupling. However, as it was shown in [27] for the 2HDM, it is possible to have large values of the cross section for very large values of the charged Higgs boson mass. Hence, this process is particularly interesting in order to investigate the mass region near the threshold (notice once more we are considering only light charged Higgs bosons, that is, with masses below the $t b$ threshold).

The most recent parton level analysis for $p p(g g+b \bar{b}) \rightarrow W^{+} H^{-}+H^{+} W^{-}$was performed in [28]. They have analysed the process $p p(b \bar{b}) \rightarrow W^{+} H^{-}$, which has a phase space similar to the gluon initiated process when the triangle diagram dominates, followed by $H^{ \pm} \rightarrow \tau \nu$. They have considered all possible decays of the $W^{ \pm}$boson. As compared to previous studies, they have now a more complete set of backgrounds that 

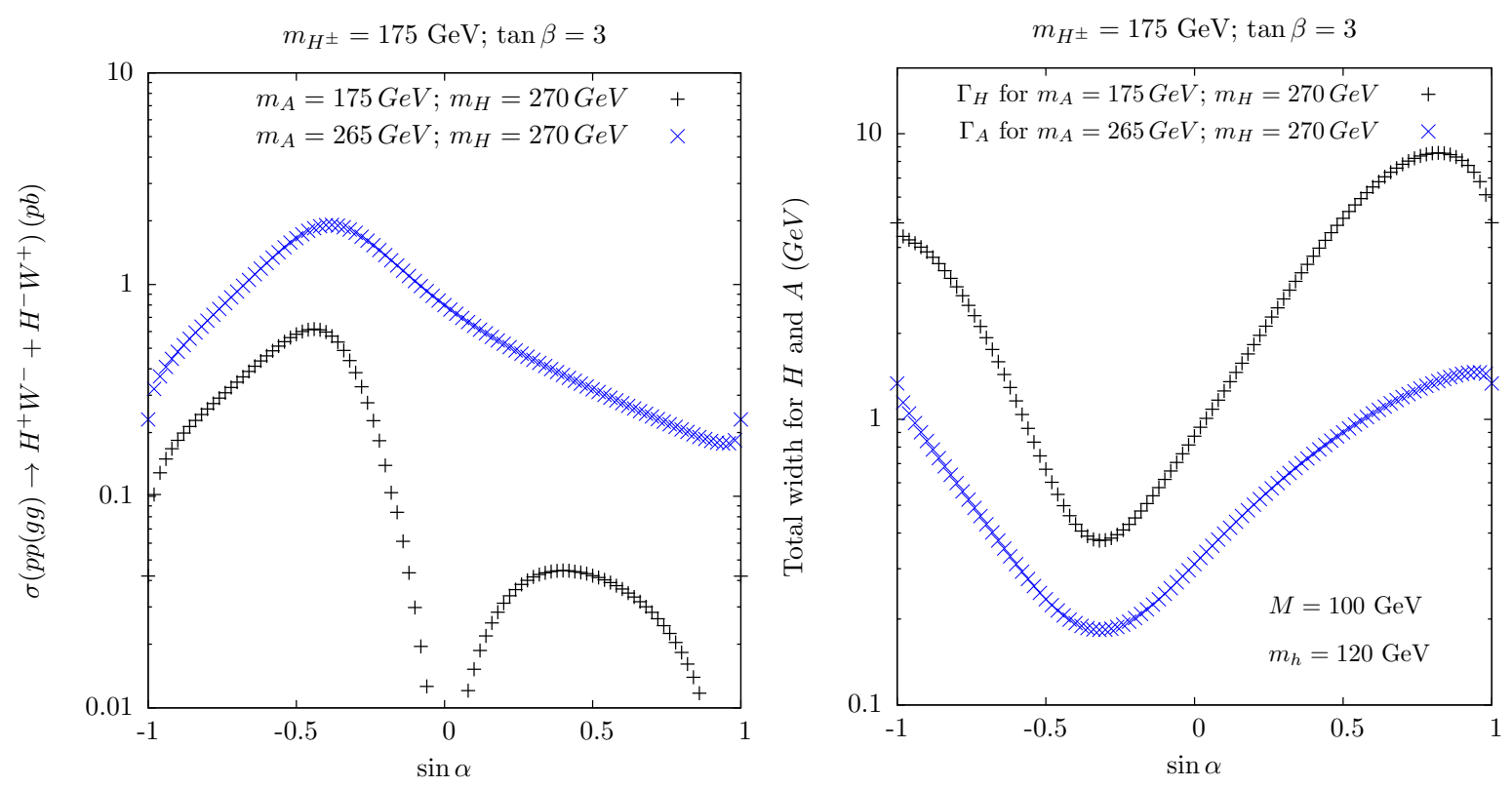

FIG. 13: In the left panel we show the production cross section for $p p(g g) \rightarrow H^{+} W^{-}+H^{-} W^{+}$as a function of $\sin \alpha$ for $m_{H^{ \pm}}=175 \mathrm{GeV}$ and $\tan \beta=3$ (the remaining set of parameters is shown in the figures). In the right panel we present the total width of the heaviest CP even Higgs boson of the CP-odd Higgs boson for the same values of the model parameters.

include $t \bar{t}, W^{+} W^{-}$and $W^{ \pm}+$jets. Combining the analysis for the leptonic $W^{ \pm}$decay and hadronic $W^{ \pm}$decay cases, we conclude that a cross section of $1 \mathrm{pb}$ can be probed with approximately $6 \mathrm{fb}^{-1}$ of integrated luminosity and $\sqrt{s}=14 \mathrm{TeV}$, for a charged Higgs mass of $175 \mathrm{GeV}$. We note however that, even with such a large cross section, the value of $S / B$ is close to $5 \%$. Nonetheless, the study presented in 28] clearly motivates a full simulation at detector level by the ATLAS and the CMS collaborations which would help to probe the region of charged Higgs masses near the threshold in these models.

In the left panel of figure 13 we show the production cross section for $p p(g g) \rightarrow H^{+} W^{-}+H^{-} W^{+}$as a function of $\sin \alpha$ for $m_{H^{ \pm}}=175 \mathrm{GeV}$ and $\tan \beta=3$ (the remaining set of parameters is shown in the figures). In the right panel we present the total width of the heaviest CP-even Higgs boson for the same values of the model parameters. First we note that, again, we have reached values of the cross sections of the level of the $p b$ with all constraints taken into account. The numbers become smaller if we move to larger values of $\tan \beta$. The values of the total width in the right panel show us how sensitive the cross section is to the width of the CP-even Higgs state, $H$, and especially to that of the CP-odd one, $A$. As discussed for charged Higgs boson pair production, the values of the cross sections are extremely sensitive to the width of the resonant particle. This process has this interesting feature of involving the CP-odd Higgs state $A$ and the fact that in some regions of the parameter space the resonant $H$ and the resonant $A$ contributions are easily distinguishable. In fact, because the CP-odd state is not allowed to decay to a gauge boson pair, its width is always much smaller than that of a scalar state with same mass, thus making resonant production via an $A$ state the largest one. Taking the heaviest $\mathrm{CP}$-even as an example, both couplings $g_{H W^{ \pm} W^{\mp}}$ and $g_{H W^{ \pm} H^{\mp}}$ are proportional to $\cos (\beta-\alpha)$ and therefore it is not possible to make one of them large and the other small simultaneously. In contrast, in the CP-odd case, the decay $A \rightarrow W^{ \pm} H^{\mp}$ is usually the largest [37] in most of the parameter space as long as it is kinematically allowed.

\section{CONCLUSIONS}

In this work we have analysed all possible search modes for a light charged Higgs boson from a 2HDM at the LHC. We have started with processes that depend only on the charged Higgs boson mass and on $\tan \beta$. Using the ATLAS and CMS studies for $p p \rightarrow t \bar{t} \rightarrow b \bar{b} W^{ \pm} H^{\mp}$ we have drawn 95\% CL exclusion 
plots in the $\left(\tan \beta, m_{H^{ \pm}}\right)$plane. For $30 \mathrm{fb}^{-1}$ of collected luminosity, the exclusion range spans from $\tan \beta \lesssim 11$ for $m_{H^{ \pm}}=90 \mathrm{GeV}$ to $\tan \beta \lesssim 6$ for $m_{H^{ \pm}}=150 \mathrm{GeV}$ in model Type $\mathrm{X}$ and $\tan \beta \lesssim 9$ for $m_{H^{ \pm}}=90 \mathrm{GeV}$ to $\tan \beta \lesssim 2$ for $m_{H^{ \pm}}=150 \mathrm{GeV}$ in model Type I. By performing a parton level analysis we have showed that the single top process deserves a full detector level analysis. In fact, taking model Type $\mathrm{X}$ as an example, our parton level analysis shows that for a mass of $100 \mathrm{GeV}$ an exclusion of $\tan \beta \lesssim 5$ could be expected at $95 \%$ CL for a collected luminosity of $10 \mathrm{fb}^{-1}$ and $\tan \beta \lesssim 7$ for $30 \mathrm{fb}^{-1}$ for model Type X. Although optimistic, the results show that a full detector level analysis would be worth performing. Combining the single top analysis with the one already performed for $t \bar{t}$, we expect to increase the excluded region in the $\left(\tan \beta, m_{H^{ \pm}}\right)$plane. Finally, the last process that could contribute to improve the exclusion limits in the $\left(\tan \beta, m_{H^{ \pm}}\right)$plane is direct charged Higgs boson production. We have shown that the cross sections are unfortunately too small and fall too rapidly with $\tan \beta$. Hence, no contribution is expected to help improving the above results. In table III we present some benchmarks for the three processes that depend only on $\tan \beta$ and the charged Higgs boson mass.

\begin{tabular}{ccccccc}
\hline \hline$m_{H^{ \pm}}(\mathrm{GeV})$ & & 100 & & & 150 & \\
\hline $\tan \beta$ & 3 & 10 & 30 & 3 & 10 & 30 \\
\hline \hline$p p \rightarrow t \bar{t} \rightarrow b \bar{b} W^{ \pm} H^{\mp}$ & Yes & Yes & No & Yes & No & No \\
\hline$p p \rightarrow H^{ \pm} b j$ & Yes & HL & No & Yes & No & No \\
\hline$p p(c s) \rightarrow H^{ \pm}(+j)$ & No & No & No & No & No & No \\
\hline \hline
\end{tabular}

TABLE III: Benchmarks for the three processes that depend only on $\tan \beta$ and on the charged Higgs mass. HL stands for High Luminosity.

If a charged Higgs boson is not found at the LHC, small values of $\tan \beta$ will be excluded with the above processes, as they rely only on the Yukawa couplings. Accessing the high $\tan \beta$ region is not possible regardless of the remaining $2 \mathrm{HDM}$ parameters. There are however some regions of the high $\tan \beta$ domain that can be probed at the LHC. We have shown that charged Higgs boson pair production via gluon fusion as well as VBF can give some scope but only in the scenarios of resonant production together with an enhancement of the Higgs self-couplings, namely the coupling between charged and neutral Higgs states. Parton level studies related to these processes lead us to the conclusion that the regions of large $\sin \alpha$ and large $\tan \beta$ give rise to cross sections of the order of the $p b$ that can be probed with just a few $\mathrm{fb}^{-1}$ of integrated luminosity. In table IV we present some benchmarks for the three processes where resonant production is allowed.

\begin{tabular}{ccccccc}
\hline \hline$m_{H^{ \pm}}(\mathrm{GeV})$ & \multicolumn{3}{c}{100} & & \multicolumn{3}{c}{150} \\
\hline $\tan \beta$ & 3 & 10 & 30 & 3 & 10 & 30 \\
\hline \hline$g g, b \bar{b}, q \bar{q} \rightarrow H^{+} H^{-}$ & Yes* $^{*}$ & Yes* & Yes* & Yes* & Yes* & Yes* \\
\hline$q Q \rightarrow q^{\prime} Q^{\prime} H^{+} H^{-}$ & Yes* $^{*}$ & Yes* & Yes* & Yes* $^{*}$ & Yes $^{*}$ & Yes $^{*}$ \\
\hline$g g, b \bar{b} \rightarrow H^{ \pm} W^{\mp}$ & Yes* $^{*}$ & HL $^{*}$ & No & Yes* & HL & No \\
\hline \hline
\end{tabular}

TABLE IV: Resonant production with enhancement of couplings. HL stands for High Luminosity. (*In definite regions of the parameter space where resonant production is allowed.)

In conclusion, if one dismisses the usual presumption that a $2 \mathrm{HDM}$ can only be motivated within Supersymmetry, thereby shifting the attention from its Type II realisation to other types, specifically to this work to the case of Types I and X, one would find interesting phenomenology emerging at the current LHC, manifesting itself in production and/or decay modes of light charged Higgs boson states, i.e., below the top mass, that are possible neither in a Type II nor in a Type Y (the latter also known as III) scenario. Very little luminosity may be necessary to ascertain the presence of such states at the CERN proton-proton accelerator running at design energy $(14 \mathrm{TeV})$ in a variety of novel signatures. In this paper, we have laid the basis for a systematic exploration of such 2HDM types in the quest for the ultimate understanding of the mechanism of EWSB. Sophisticated experimental analyses are now needed in order to finally confirm or disprove the validity of these $2 \mathrm{HDM}$ hypotheses. To this end, we have produced computational tools, selection procedures and benchmark scenarios that can readily be exploited in the LHC environment. 
Acknowledgements We thank Nuno Castro and Filipe Veloso for discussions. We thank Oliver Brein for checking cross sections of gluon fusion processes. All authors acknowledge financial support from the PMI2 Connect Initiative in the form of a Research Cooperation Grant. SM is partially supported by the NExT Institute. RG is supported by a Fundação para a Ciência e Tecnologia Grant SFRH/BPD/47348/2008. MA is supported in part by Grant-in-Aid for Young Scientists (B) no. 22740137. SK is supported in part by Grant-in-Aid for Scientific Research (A) no. 22244031 and (C) no. 19540277. KY is supported by the Japanese Society for the Promotion of Science (JSPS Fellow (DC2)). RS is partially supported by an FP7 Reintegration Grant, number PERG08-GA-2010-277025. RG and RS are partially supported by PEst-OE/FIS/UI0618/2011.

\section{Appendix A: Parton level analysis of charged Higgs production via single top production}

In this Alppendix we present the highlights of the parton level analysis for the single top case. As mentioned before we take as our signal the process

$$
p p \rightarrow H^{ \pm} b j \rightarrow b j \tau \nu_{\tau} .
$$

Regarding the background processes we consider the irreducible background processes

$$
p p \rightarrow \operatorname{bjl}(\tau, \mu, e) \nu_{\tau, \mu, e}
$$

and the reducible one

$$
p p \rightarrow t \bar{t} \rightarrow W^{+} b W^{-} \bar{b}
$$

where both $W^{ \pm}$'s can decay semi-leptonic, fully leptonic or fully hadronic.
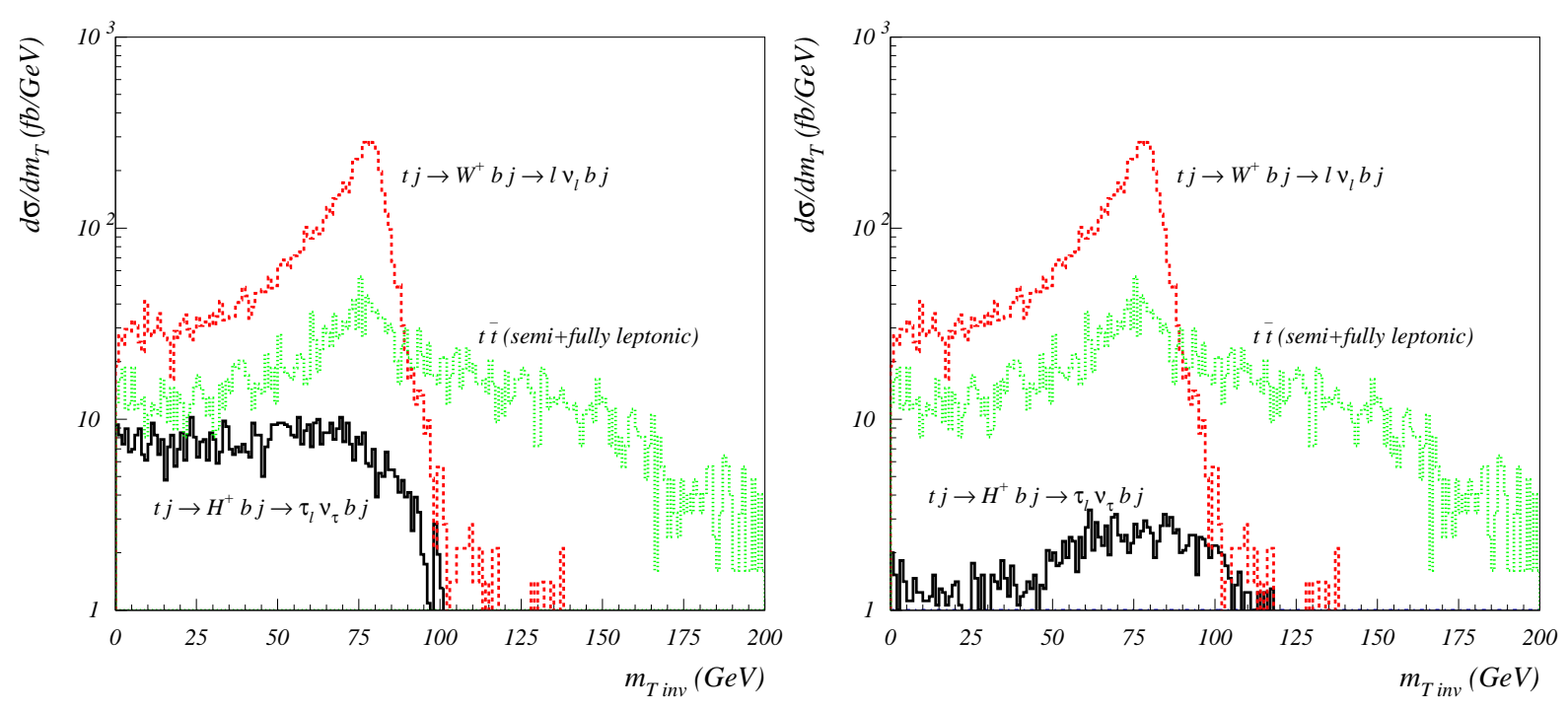

FIG. 14: In the left panel we show the transverse mass distribution for $m_{H^{ \pm}}=100 \mathrm{GeV}$ and tan $\beta=1.5$ for signal and backgrounds. In the right panel we present the the same plot but for a charged Higgs boson mass of $m_{H^{ \pm}}=140 \mathrm{GeV}$.

First we have to eliminate the huge reducible background coming from $p p \rightarrow t \bar{t}$. We will consider processes with at least one lepton which means that we exclude the fully hadronic $t \bar{t}$ production background. The fully hadronic background is almost completely rejected by asking for a lepton with transverse momentum $p_{T}$ greater than $30 \mathrm{GeV}$ in the central detector region of $|\eta| \leq 2.5$. The fully leptonic background is rejected by applying a veto on events that contain a second lepton with transverse momentum above $10 \mathrm{GeV}$. These are the strategies developed in [56] for SM single top production, which shows that the 
fully hadronic background can easily be reduced but should nevertheless be taken into account in a full detector level analysis. The semi-leptonic contribution is the hardest reducible background to deal with. In order to reduce it we apply a veto on events with more than two jets with transverse momentum greater than $15 \mathrm{GeV}$. This reduces the background to levels that are below the ones presented in 56 ] because we have no jets coming from hadronisation as this is a parton level analysis. Therefore, we have estimated in our semileptonic $t \bar{t}$ background a reduction of a factor of 2 as compared to Ref. [56] where, due the hadronisation of the jets, a veto is applied on events with more than four jets with transverse momentum greater than $15 \mathrm{GeV}$. Therefore our estimate of the total reducible background is probably optimistic by a factor of approximately 1.4.

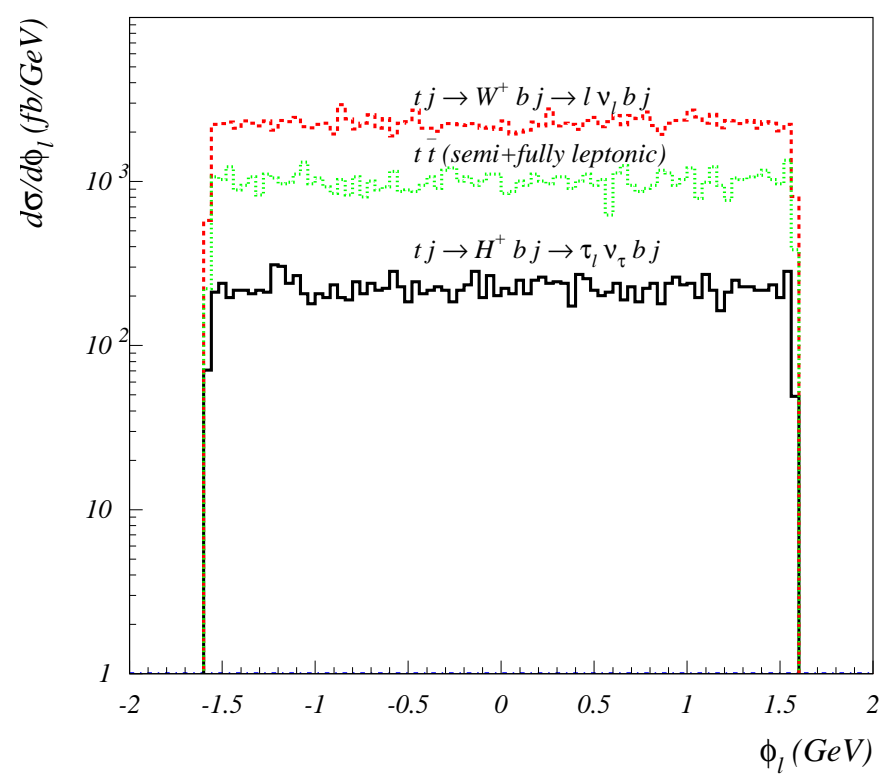

FIG. 15: Azimuthal angle distribution for $m_{H^{ \pm}}=100 \mathrm{GeV}$ and $\tan \beta=1.5$ for signal and backgrounds.

Regarding the irreducible single top background we have looked at several distributions in order to find suitable cuts to minimise its effects. As expected, all $p_{T}$ distributions (of the lepton or any of the jets in the process) are very similar for both signal and irreducible background. The same is true for the missing energy distribution. Our hopes relied mainly on the lepton angular distributions (because of the different chirality in the couplings of the $W^{ \pm}$and $H^{ \pm}$bosons with the leptons) and on the transverse mass distribution as defined in [57]

$$
M_{T}(l \nu)=\sqrt{2\left|p_{T}^{l}\right|\left|p_{T}^{\text {miss }}\right|-2 \vec{p}_{T}^{l} \cdot \vec{p}_{T}^{\text {miss }}}
$$

where the superscript $l$ refers to electron and/or muon and the superscript "miss" refers to total missing energy and total missing transverse momentum. In the left (right) panel of figure 14 we present the transverse mass distribution for $m_{H^{ \pm}}=100(140) \mathrm{GeV}$ and $\tan \beta=1.5$ for signal and backgrounds. It is clear that we can reduce drastically the background by avoiding the large transverse mass region. Therefore, we apply the following cut on the transverse mass, $m_{T}<50 \mathrm{GeV}$, which optimises the significance for a $100 \mathrm{GeV}$ Higgs boson. As the mass of the charged Higgs boson grows, this cut becomes less efficient because the signal maximum values move towards the $W^{ \pm}$mass peak as can be clearly seen in the right panel of figure 14. Finally, all angular distributions related to the lepton have shown to be of no use in improving the analysis. As an example, we plot in figure 15 the azimuthal angle distribution of the lepton (for $m_{H^{ \pm}}=100 \mathrm{GeV}$ and $\tan \beta=1.5$ ). We believe that, because the leptons are highly boosted, the potential differences in the lepton angular distributions are washed out. Conversely, the presence of undetectable particles in the final state prevents one from reconstructing the reference frame of the decaying boson.

In summary, we have used the following set of cuts: 
1. $\Delta R_{j j}>0.4$ and $\Delta R_{j l}>0.2$ where $j$ stands both for light jets and $b$-jets and $l$ is a lepton (electron or muon);

2. we demand a lepton with transverse momentum greater than $p_{T}>30 \mathrm{GeV}$ in the central detector region of $|\eta| \leq 2.5$;

3. to reject dilepton events a veto is applied on events that contain a second lepton with transverse momentum above $10 \mathrm{GeV}$;

4. to reduce the QCD background, we apply a cut on the missing transverse momentum: we demand $p_{T}^{\text {miss }}>20 \mathrm{GeV}$;

5. we demand events with at least two jets to have transverse momentum greater than $30 \mathrm{GeV}$ and to be in the region of the detector with $|\eta| \leq 4.5$;

6. a veto is applied on events with more than two jets with transverse momentum greater than 15 $\mathrm{GeV}$;

7. we apply the following cut on the transverse mass: $m_{T}<50 \mathrm{GeV}$;

8. the efficiency to identify a lepton was chosen as $50 \%$;

which led to the significances and $S / B$ ratios presented in table [I We end this Appendix with a final comment on another important background, $W^{ \pm}+n$ jets, which was shown by [56] to be negligible when the above set of cuts are imposed. The $W^{ \pm}+n$ jets background has a cross section two orders of magnitude larger than the $t \bar{t}$ one. If the $W^{ \pm}$decays hadronically this is just like all other QCD backgrounds and we can use a lepton and the missing transverse energy to discriminate against events containing only jets. If the $W^{ \pm}$boson decays leptonically, we can reject about $95 \%$ of all remaining $W^{ \pm}+n$ jets events by demanding a $b$-tagged jet (which we do by demanding a $b$-jet with a transverse energy larger than $30 \mathrm{GeV}$ ). According to [56], the $W^{ \pm}+j e t s$ background should be about $10 \%$ of the total background in the case of SM single top production.

\section{Appendix B: Charged Higgs boson pair production in left-right symmetric models}

A study of charged Higgs pair production in the context of a left-right symmetric model was first performed in [45]. A particular final state, $e^{ \pm} \mu^{\mp}+\not p_{T}$, was chosen and a significance of $\sigma_{S} / \sqrt{\sigma_{B}} \approx 1.2 \mathrm{fb} b^{1 / 2}$ was obtained, for a charged Higgs boson mass of $100 \mathrm{GeV}$. The large value for the signal cross section is mainly due to the values of the BRs - in these models the charged Higgs boson decays to a lepton and an anti-neutrino independently of the lepton family. More recently, see [46], a similar study was performed in the context of a 2HDM-like model, where three gauge-singlet right-handed Weyl spinors were added to become the right-handed components of the three Dirac neutrinos. The authors of this paper have investigated three possible final states, $e^{+} e^{-}+\not p_{T}, \mu^{+} \mu^{-}+\not p_{T}$ and $e^{ \pm} e^{\mp}+\not p_{T}$, for charged Higgs boson masses of 100 and $300 \mathrm{GeV}$ and in the scenario where $\mathrm{BR}\left(H^{+} \rightarrow e^{+} \nu\right)=\mathrm{BR}\left(H^{+} \rightarrow \mu^{+} \nu\right)=1 / 3$. Combining all analyses for this particular scenario they conclude that a $20 \mathrm{fb}^{-1}$ luminosity is needed for a discovery with a $5 \sigma$ significance for a $100 \mathrm{GeV}$ charged Higgs boson. We will now use their results to make an estimate of the cross sections that can be probed in both models Type I and X. Before proceeding we should however note that these are truly crude estimates, i.e., although the final states are the same and the production process looks similar, there are differences due to the structure of the couplings, e.g., it is clear that the amount of missing energy present, that would reflect in the signal efficiency, is different in each case. Nevertheless we believe we can use their results as a guide until a parton level analysis for this specific process is available. Finally, before presenting their results applied to our models, we should mention that the final states chosen in [45, 46] are not the ones that would give the largest number of signal events. In fact, taking into account the values for the BRs presented in table $\mathrm{V}$, a final state where one $\tau$ decays leptonically and the other hadronically is obviously more appropriate to study models Type $\mathrm{I}$ and $\mathrm{X}$.

In 2 HDMs Type I and $\mathrm{X}$, and for $\tan \beta \gtrsim 2$, the main decay of the charged Higgs boson is $H^{+} \rightarrow \tau^{+} \nu$ and decays to $\mu \nu$ and $e \nu$ are negligible. Then, using the scenario in [45, 46], both $\tau$ 's have to decay 


\begin{tabular}{cccc}
\hline \hline $\tan \beta\left(m_{H^{ \pm}}=100 \mathrm{GeV}\right)$ & 1 & 3 & 30 \\
\hline \hline $\mathrm{BR}_{I}\left(H^{ \pm} \rightarrow \tau_{l} \nu\right) \times \mathrm{BR}_{I}\left(H^{ \pm} \rightarrow c s\right)$ & $8 \%$ & $8 \%$ & $8 \%$ \\
\hline $\mathrm{BR}_{I}\left(H^{ \pm} \rightarrow \tau_{l} \nu\right) \times \mathrm{BR}_{I}\left(H^{ \pm} \rightarrow \tau \nu\right)$ & $28 \%$ & $28 \%$ & $28 \%$ \\
\hline $\mathrm{BR}_{X}\left(H^{ \pm} \rightarrow \tau_{l} \nu\right) \times \mathrm{BR}_{X}\left(H^{ \pm} \rightarrow c s\right)$ & $8 \%$ & $0.2 \%$ & $\approx 0$ \\
\hline $\mathrm{BR}_{X}\left(H^{ \pm} \rightarrow \tau_{l} \nu\right) \times \mathrm{BR}_{X}\left(H^{ \pm} \rightarrow \tau \nu\right)$ & $28 \%$ & $58 \%$ & $58 \%$ \\
\hline \hline
\end{tabular}

TABLE V: Product of $\tau$ BRs for a charged Higgs boson mass of $100 \mathrm{GeV}$ in models Type I and X and for three values of $\tan \beta$. The largest numbers occur for the case when one $\tau$ decays leptonically and the other hadronically (we are considering that we need at least one $\tau$ do decay leptonically for triggering purposes).

leptonically to an electron or a muon plus missing energy with SM rates. The number of signal events is given by

$$
\sigma\left(p p \rightarrow H^{+} H^{-}\right) \mathrm{BR}\left(H^{+} \rightarrow \tau^{+} \nu\right) \mathrm{BR}\left(H^{-} \rightarrow \tau^{-} \nu\right) \mathrm{BR}\left(\tau^{+} \rightarrow l^{+} \nu\right) \mathrm{BR}\left(\tau^{-} \rightarrow l^{\prime-} \nu\right)
$$

where $l, l^{\prime}=\mu, e$. The $\operatorname{BR}\left(\tau^{+} \rightarrow l^{+} \nu\right)$ is $\approx 17 \%$ for muons and $\approx 18 \%$ for electrons. For $\tan \beta \gtrsim 2$, $\mathrm{BR}\left(H^{+} \rightarrow \tau^{+} \nu\right)$ is $69 \%$ in model Type I and close to $100 \%$ in model Type $\mathrm{X}$. Taking the previous studies [45, 46] as a guide, we would conclude that a significant cross section is one of the order of 400 $f b$. This means that, using their analysis for our signal despite the difference in, e.g., the total missing energy (note that the background is the same), one would be able to start probing a significant parameter region with $30 \mathrm{fb}^{-1}$ for $2 \mathrm{HDM}$ Type $\mathrm{X}$ if the production cross section was of the order of $400 \mathrm{fb}$.

[1] LEP Higgs Working Group for Higgs boson searches, ALEPH, DELPHI,L3 and OPAL Collaborations, arXiv: hep-ex/0107031

[2] H. E. Logan and D. MacLennan, Phys. Rev. D 79 (2009) 115022.

[3] P. Gutierrez [CDF and D0 Collaborations], FERMILAB-CONF-10-540-E; V. M. Abazov et al. [D0 Collaboration], Phys. Rev. D 80 (2009) 051107; CDF Collaboration, CDF/PUB/TOP/PUBLIC/7712.

[4] V. D. Barger, J. L. Hewett and R. J. N. Phillips, Phys. Rev. D 41 (1990) 3421.

[5] M. Aoki, S. Kanemura, K. Tsumura and K. Yagyu, Phys. Rev. D 80 (2009) 015017.

[6] W. S. Hou and R. S. Willey, Phys. Lett. B 202 (1988) 591; M. Ciuchini, G. Degrassi, P. Gambino and G. F. Giudice, Nucl. Phys. B 527 (1998) 21; F. Borzumati and C. Greub, Phys. Rev. D 58, 074004 (1998); F. Borzumati and C. Greub, Phys. Rev. D 59, 057501 (1999); C. Amsler et al., Phys. Lett. B 667 (2008) 1; The Belle Collaboration, Phys. Rev. Lett. 103 (2009) 241801.

[7] S. Su, B. Thomas, Phys. Rev. D79, 095014 (2009). arXiv:0903.0667 [hep-ph]]

[8] M. Aoki, S. Kanemura and O. Seto, Phys. Rev. Lett. 102 (2009) 051805; M. Aoki, S. Kanemura and O. Seto, Phys. Rev. D 80 (2009) 033007; M. Aoki, S. Kanemura and K. Yagyu, arXiv:1102.3412 [hep-ph]]; H. S. Goh, L. J. Hall and P. Kumar, JHEP 0905 (2009) 097.

[9] S. Kanemura, Y. Okada, E. Senaha and C. P. Yuan, Phys. Rev. D 70 (2004) 115002.

[10] S. L. Glashow and S. Weinberg, Phys. Rev. D 15, 1958 (1977).

[11] Y. Grossman, Nucl. Phys. B 426, 355 (1994).

[12] A. G. Akeroyd and W. J. Stirling, Nucl. Phys. B 447 (1995) 3.

[13] A. Denner, R. J. Guth, W. Hollik and J. H. Kuhn, Z. Phys. C 51 (1991) 695.

[14] K. Nakamura et al. [Particle Data Group], J. Phys. G 37 (2010) 075021.

[15] A. Wahab El Kaffas, P. Osland and O. Magne Ogreid, Phys. Rev. D 76 (2007) 095001.

[16] M. Krawczyk and J. Zochowski, Phys. Rev. D 55 (1997) 6968; A. Dedes and H. E. Haber, JHEP 0105 (2001) 006; K. Cheung, C. H. Chou and O. C. W. Kong, Phys. Rev. D 64 (2001) 111301; K. Cheung and O. C. W. Kong, Phys. Rev. D 68 (2003) 053003; F. Jegerlehner and A. Nyffeler, Phys. Rept. 477 (2009) 1.

[17] S. Kanemura, T. Kubota and E. Takasugi, Phys. Lett. B 313 (1993) 155; A. G. Akeroyd, A. Arhrib and E. M. Naimi, Phys. Lett. B 490 (2000) 119; J. Horejsi and M. Kladiva, Eur. Phys. J. C 46 (2006) 81.

[18] N. G. Deshpande and E. Ma, Phys. Rev. D 18 (1978) 2574.

[19] P. M. Ferreira, R. Santos and A. Barroso, Phys. Lett. B 603 (2004) 219 [Erratum-ibid. B 629 (2005) 114]; M. Maniatis, A. von Manteuffel, O. Nachtmann and F. Nagel, Eur. Phys. J. C 48 (2006) 805; I. P. Ivanov, Phys. Rev. D 75 (2007) 035001 [Erratum-ibid. D 76 (2007) 039902]; A. Barroso, P. M. Ferreira and R. Santos, Phys. Lett. B 632 (2006) 684.

[20] J. F. Gunion, H. E. Haber, F. E. Paige, W. K. Tung and S. S. D. Willenbrock, Nucl. Phys. B 294 (1987) 621; J. L. Diaz-Cruz and O. A. Sampayo, Phys. Rev. D 50 (1994) 6820; S. Moretti and D. P. Roy, Phys. Lett. B 470 (1999) 209; D. J. . Miller, S. Moretti, D. P. Roy and W. J. Stirling, Phys. Rev. D 61 (2000) 055011. 
[21] S. Moretti and K. Odagiri, Phys. Rev. D 55 (1997) 5627.

[22] H. J. He and C. P. Yuan, Phys. Rev. Lett. 83 (1999) 28.

[23] J. L. Diaz-Cruz, H. J. He and C. P. Yuan, Phys. Lett. B 530 (2002) 179 arXiv:hep-ph/0103178.

[24] S. R. Slabospitsky, arXiv:hep-ph/0203094.

[25] S. Dittmaier, G. Hiller, T. Plehn and M. Spannowsky, Phys. Rev. D 77 (2008) 115001.

[26] D. A. Dicus, J. L. Hewett, C. Kao and T. G. Rizzo, Phys. Rev. D 40 (1989) 787; A. A. Barrientos Bendezu and B. A. Kniehl, Phys. Rev. D 59 (1999) 015009; S. Moretti and K. Odagiri, Phys. Rev. D 59 (1999) 055008; A. A. Barrientos Bendezu and B. A. Kniehl, Phys. Rev. D 61 (2000) 097701; O. Brein, W. Hollik and S. Kanemura, Phys. Rev. D 63 (2001) 095001 arXiv:hep-ph/0008308.

D. Eriksson, S. Hesselbach and J. Rathsman, Eur. Phys. J. C 53 (2008) 267; W. Hollik and S. h. Zhu, Phys. Rev. D 65 (2002) 075015.

[27] E. Asakawa, O. Brein and S. Kanemura, Phys. Rev. D 72 (2005) 055017.

[28] M. Hashemi, arXiv:1008.3785 [hep-ph].

[29] S. Kanemura and C. P. Yuan, Phys. Lett. B 530 (2002) 188; A. G. Akeroyd, Phys. Rev. D 68 (2003) 077701; A. G. Akeroyd and M. A. Diaz, Phys. Rev. D 67 (2003) 095007; Q. H. Cao, S. Kanemura and C. P. Yuan, Phys. Rev. D 69 (2004) 075008; A. Belyaev, Q. H. Cao, D. Nomura, K. Tobe and C. P. Yuan, Phys. Rev. Lett. 100 (2008) 061801; X. Miao, S. Su and B. Thomas, Phys. Rev. D 82 (2010) 035009;

[30] S. S. D. Willenbrock, Phys. Rev. D 35 (1987) 173; Y. Jiang, L. Han, W. G. Ma, Z. H. Yu and M. Han, J. Phys. G 23 (1997) 385; A. Krause, T. Plehn, M. Spira and P. M. Zerwas, Nucl. Phys. B 519 (1998) 85; A. A. Barrientos Bendezu and B. A. Kniehl, Nucl. Phys. B 568 (2000) 305; O. Brein and W. Hollik, Eur. Phys. J. C 13 (2000) 175;

[31] A. Alves and T. Plehn, Phys. Rev. D 71 (2005) 115014.

[32] E. Eichten, I. Hinchliffe, K. D. Lane and C. Quigg, Rev. Mod. Phys. 56 (1984) 579 [Addendum-ibid. 58 (1986) $1065]$.

[33] S. Moretti, J. Phys. G 28 (2002) 2567.

[34] E. W. N. Glover and J. J. van der Bij, Nucl. Phys. B 309 (1988) 282; D. A. Dicus, C. Kao and S. S. D. Willenbrock, Phys. Lett. B 203 (1988) 457; G. D. Kribs, T. Plehn, M. Spannowsky and T. M. P. Tait, Phys. Rev. D 76 (2007) 075016; T. Plehn, M. Spira and P. M. Zerwas, Nucl. Phys. B 479 (1996) 46 [Erratum-ibid. B 531 (1998) 655]; U. Baur, T. Plehn and D. L. Rainwater, Phys. Rev. D 67 (2003) 033003, Phys. Rev. D 68 (2003) 033001 and Phys. Rev. D 69 (2004) 053004; J. Dai, J. F. Gunion and R. Vega, Phys. Lett. B 371 (1996) 71 and Phys. Lett. B 387 (1996) 801; M. Moretti, S. Moretti, F. Piccinini, R. Pittau and J. Rathsman, JHEP 0712 (2007) 075 and JHEP 1011 (2010) 097; M. Moretti, S. Moretti, F. Piccinini, R. Pittau and A. D. Polosa, JHEP 0502 (2005) 024 and arXiv:hep-ph/0411039; S. Moretti and J. Rathsman, Eur. Phys. J. C 33 (2004) 41.

[35] M. Guchait and S. Moretti, JHEP 0201 (2002) 001; K. A. Assamagan, M. Guchait and S. Moretti, arXiv:hep-ph/0402057; J. Alwall and J. Rathsman, JHEP 0412 (2004) 050; T. Plehn, Phys. Rev. D 67 (2003) 014018; S. h. Zhu, Phys. Rev. D 67 (2003) 075006; J. Alwall, C. Biscarat, S. Moretti, J. Rathsman and A. Sopczak, Eur. Phys. J. direct C39S1 (2005) 37.

[36] A. Arhrib, R. Benbrik, C. H. Chen, R. Guedes and R. Santos, JHEP 0908 (2009) 035.

[37] S. Kanemura, S. Moretti, Y. Mukai, R. Santos and K. Yagyu, Phys. Rev. D 79 (2009) 055017.

[38] A. Akeroyd, M. Aoki, R. Guedes, S. Kanemura, S. Moretti, R. Santos and K. Yagyu, in preparation.

[39] J. Kalinowski and M. Krawczyk, Phys. Lett. B 361 (1995) 66; A. Belyaev, R. Guedes, S. Moretti and R. Santos, Phys. Rev. D 81 (2010) 095006.

[40] S. Schael et al. [ALEPH, DELPHI, L3 and OPAL Collaborations], Eur. Phys. J. C 47 (2006) 547.

[41] G. Aad et al. [The ATLAS Collaboration], arXiv:0901.0512 [hep-ex]; K. A. Assamagan, Y. Coadou and A. Deandrea, Eur. Phys. J. direct C 4 (2002) 9.

[42] CMS Collaboration, Physics Technical Design Report, Volume 2. CERN/LHCC 2006- 021.

[43] A. Pukhov, arXiv:hep-ph/0412191.

[44] J. Alwall et al., JHEP 0709 (2007) 028.

[45] A. Datta and A. Raychaudhuri, Phys. Rev. D 62 (2000) 055002 arXiv:hep-ph/9905421.

[46] S. M. Davidson and H. E. Logan, arXiv:1009.4413 [hep-ph].

[47] T. Hahn, Comput. Phys. Commun. 140 (2001) 418; T. Hahn, C. Schappacher, Comput. Phys. Commun. 143 (2002) 54; J. Küblbeck, M. Böhm, A. Denner, Comput. Phys. Commun. 60 (1990) 165.

[48] T. Hahn and J. I. Illana, arXiv:0708.3652 [hep-ph]. T. Hahn and J. I. Illana, Nucl. Phys. Proc. Suppl. 160 (2006) 101; T. Hahn, M. Perez-Victoria, Comput. Phys. Commun. 118 (1999) 153.

[49] G. J. van Oldenborgh, Comput. Phys. Commun. 66 (1991) 1; T. Hahn, Acta Phys. Polon. B 30 (1999) 3469.

[50] J. Pumplin, A. Belyaev, J. Huston, D. Stump and W. K. Tung, JHEP 0602 (2006) 032.

[51] F. Cornet and W. Hollik, Phys. Lett. B 669 (2008) 58.

[52] A. Arhrib, R. Benbrik, C. H. Chen and R. Santos, Phys. Rev. D 80 (2009) 015010.

[53] A. Belyaev, R. Guedes, S. Moretti and R. Santos, JHEP 1007 (2010) 051.

[54] S. Kanemura, S. Moretti and K. Odagiri, arXiv:hep-ph/0101354.

[55] S. Kanemura, S. Moretti and K. Odagiri, JHEP 0102 (2001) 011.

[56] C. Lange, Diploma thesis, Humboldt Universitt zu Berlin, January 2009

[57] V. D. Barger, T. Han and R. J. N. Phillips, Phys. Rev. D 36 (1987) 295. 